Document downloaded from:

http://hdl.handle.net/10251/63409

This paper must be cited as:

Collaboration TA, G. Bertone DH and JS, Schumann M, et al. Search of dark matter annihilation in the galactic centre using the ANTARES neutrino telescope. J Cosmol Astropart Phys. 2015;2015(10):068-068. doi:10.1088/1475-7516/2015/10/068.

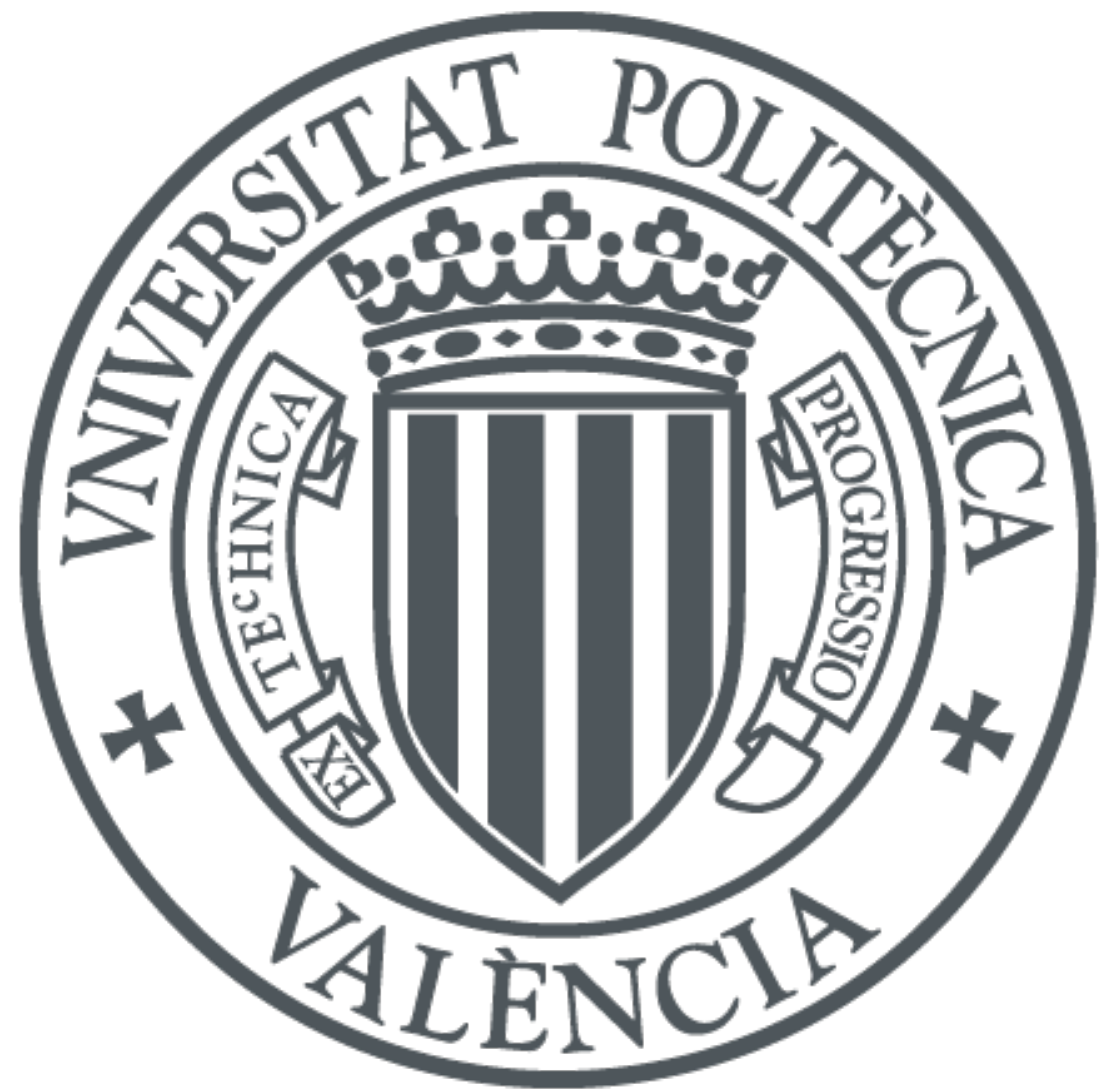

The final publication is available at

http://dx.doi.org/10.1088/1475-7516/2015/10/068

Copyright IOP Publishing: Hybrid Open Access

Additional Information 


\section{Search of Dark Matter Annihilation in the Galactic Centre using the ANTARES Neutrino Telescope}

S. Adrián-Martínez ${ }^{a}$ A. Albert ${ }^{b}$ M. Andréc G. Anton ${ }^{d}$ M. Ardid ${ }^{a}$ J.-J. Aubert ${ }^{e}$ B. Baret ${ }^{f}$ J. Barrios-Martí ${ }^{g}$ S. Basa ${ }^{h}$ V. Bertin ${ }^{e}$ S. Biagi ${ }^{i, j}$ C. Bogazzi ${ }^{k}$ R. Bormuth ${ }^{k, l}$ M. Bou-Cabo ${ }^{a}$ M.C. Bouwhuis ${ }^{k}$ R. Bruijn ${ }^{k, m}$ J. Brunner ${ }^{e}$ J. Busto ${ }^{e}$ A. Capone ${ }^{n, o}$ L. Caramete ${ }^{p}$ J. Carr $^{e}$ T. Chiarusi ${ }^{i}$ M. Circella ${ }^{q}$ R. Coniglione ${ }^{r}$ H. Costantini ${ }^{e}$ P. Coyle ${ }^{e}$ A. Creusot ${ }^{f}$ I. Dekeyser ${ }^{s}$ A. Deschamps ${ }^{t}$ G. De Bonis ${ }^{n, o}$ C. Distefano ${ }^{r}$ C. Donzaud ${ }^{f, u}$ D. Dornic ${ }^{e}$ D. Drouhin ${ }^{b}$ A. Dumas ${ }^{v}$ T. Eberl ${ }^{d}$ D. Elsässer ${ }^{w}$ A. Enzenhöfer ${ }^{d}$ K. Fehn ${ }^{d}$ I. Felis ${ }^{a}$ P. Fermani ${ }^{n, o}$ F. Folger ${ }^{d}$ L.A. Fusco ${ }^{i, j}$ S. Galatà ${ }^{f}$ P. Gay ${ }^{v}$ S. Geißelsöder ${ }^{d}$ K. Geyer ${ }^{d}$ V. Giordano ${ }^{x}$ A. Gleixner ${ }^{d}$ R. Gracia-Ruiz ${ }^{f}$ K. Graf $^{d}$ H. van Haren ${ }^{z}$ A.J. Heijboer ${ }^{k}$ Y. Hello ${ }^{t}$ J.J. Hernández- $\operatorname{Rey}^{g}$ A. Herrero ${ }^{a}$ J. Hößl ${ }^{d}$ J. Hofestädt ${ }^{d}$ C. Hugon ${ }^{a a, a b}$ C.W James ${ }^{d}$ M. de Jong ${ }^{k, l}$ M. Kadler ${ }^{w}$ O. Kalekin ${ }^{d}$ U. Katz ${ }^{d}$ D. Kießling ${ }^{d}$ P. Kooijman ${ }^{k, a c, m}$ A. Kouchner ${ }^{f}$ I. Kreykenbohm ${ }^{a d}$ V. Kulikovskiy ${ }^{a e, a}$ R. Lahmann $^{d}$ G. Lambard ${ }^{g}$ D. Lattuada ${ }^{r}$ D. Lefèvre ${ }^{s}$ E. Leonora ${ }^{a b, a f}$ S. Loucatos ${ }^{a g}$ M. Marcelin ${ }^{h}$ A. Margiotta ${ }^{i, j}$ J.A. Martínez-Mora ${ }^{a}$ S. Martini ${ }^{s}$ A. Mathieu ${ }^{e}$ T. Michael ${ }^{k}$ P. Migliozzi ${ }^{a h}$ A. Moussa ${ }^{y}$ C. Mueller ${ }^{a d}$ M. Neff ${ }^{d}$ E. Nezrì ${ }^{h}$ G.E. Păvălaș ${ }^{p}$ C. Pellegrino ${ }^{i, j}$ C. Perrina ${ }^{n, o}$ P. Piattelli ${ }^{r}$ V. Popa $^{p}$ T. Pradier ${ }^{a i}$ C. Racca ${ }^{b}$ G. Riccobene ${ }^{r}$ R. Richter ${ }^{d}$ K. Roensch ${ }^{d}$ A. Rostovtsev ${ }^{a j}$ M. Saldaña ${ }^{a}$ D. F. E. Samtleben ${ }^{k, l}$ M. Sanguineti ${ }^{a, a b}$ P. Sapienza ${ }^{r}$ J. Schmid ${ }^{d}$ J. Schnabel ${ }^{d}$ S. Schulte ${ }^{k}$ F. Schüssler ${ }^{a g}$ T. Seitz ${ }^{d}$ C. Sieger ${ }^{d}$ M. Spurio ${ }^{i, j}$ J.J.M. Steijger ${ }^{k}$ Th. Stolarczyk $^{a g}$ A. Sánchez-Losa ${ }^{g}$ M. Taiuti ${ }^{a a, a b}$ C. Tamburini ${ }^{s}$ A. Trovato ${ }^{r}$ M. Tselengidou ${ }^{d}$ C. Tönnis ${ }^{g}$ B. Vallage ${ }^{a g}$ C. Vallée ${ }^{e}$ V. Van Elewyck ${ }^{f}$ E. Visser ${ }^{k}$ D. Vivolo ${ }^{a h, a k}$ S. Wagner ${ }^{d}$ J. Wilms ${ }^{a d}$ J.D. Zornoza ${ }^{g}$ J. Zúñiga ${ }^{g}$

\footnotetext{
${ }^{a}$ Institut d'Investigació per a la Gestió Integrada de les Zones Costaneres (IGIC) - Universitat Politècnica de València. C/ Paranimf 1, 46730 Gandia, Spain.
} 
${ }^{b}$ GRPHE - Université de Haute Alsace - Institut universitaire de technologie de Colmar, 34 rue du Grillenbreit BP 50568 - 68008 Colmar, France

${ }^{c}$ Technical University of Catalonia, Laboratory of Applied Bioacoustics, Rambla Exposició,08800 Vilanova i la Geltrú,Barcelona, Spain

$d_{\text {Friedrich-Alexander-Universität Erlangen-Nürnberg, Erlangen Centre for Astroparticle Physics, Erwin-Rommel-Str. }}$ 1, 91058 Erlangen, Germany

${ }^{e}$ CPPM, Aix-Marseille Université, CNRS/IN2P3, Marseille, France

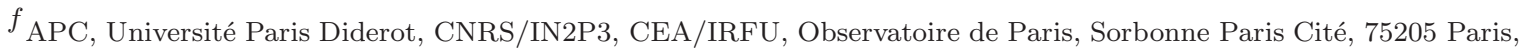
France

${ }^{g}$ IFIC - Instituto de Física Corpuscular, Edificios Investigación de Paterna, CSIC - Universitat de València, Apdo. de Correos 22085, 46071 Valencia, Spain

${ }^{h}$ LAM - Laboratoire d'Astrophysique de Marseille, Pôle de l'Étoile Site de Château-Gombert, rue Frédéric Joliot-Curie 38, 13388 Marseille Cedex 13, France

${ }^{i}$ INFN - Sezione di Bologna, Viale Berti-Pichat 6/2, 40127 Bologna, Italy

${ }^{j}$ Dipartimento di Fisica dell'Università, Viale Berti Pichat 6/2, 40127 Bologna, Italy

${ }^{k}$ Nikhef, Science Park, Amsterdam, The Netherlands

${ }^{l}$ Huygens-Kamerlingh Onnes Laboratorium, Universiteit Leiden, The Netherlands

${ }^{m}$ Universiteit van Amsterdam, Instituut voor Hoge-Energie Fysica, Science Park 105, 1098 XG Amsterdam, The Netherlands

${ }^{n}$ INFN -Sezione di Roma, P.le Aldo Moro 2, 00185 Roma, Italy

o Dipartimento di Fisica dell'Università La Sapienza, P.le Aldo Moro 2, 00185 Roma, Italy

$p$ Institute for Space Science, RO-077125 Bucharest, Măgurele, Romania

${ }^{q}$ INFN - Sezione di Bari, Via E. Orabona 4, 70126 Bari, Italy

${ }^{r}$ INFN - Laboratori Nazionali del Sud (LNS), Via S. Sofia 62, 95123 Catania, Italy

${ }^{s}$ Mediterranean Institute of Oceanography (MIO), Aix-Marseille University, 13288, Marseille, Cedex 9, France; Universit du Sud Toulon-Var, 83957, La Garde Cedex, France CNRS-INSU/IRD UM 110

${ }^{t}$ Géoazur, Université Nice Sophia-Antipolis, CNRS, IRD, Observatoire de la Côte d'Azur, Sophia Antipolis, France

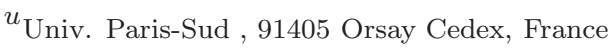

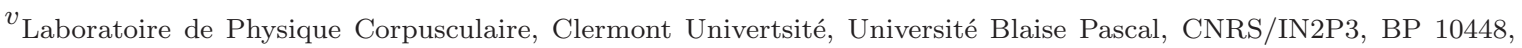
F-63000 Clermont-Ferrand, France

wInstitut für Theoretische Physik und Astrophysik, Universität Würzburg, Emil-Fischer Str. 31, 97074 Wrzburg, Germany

${ }^{x}$ INFN - Sezione di Catania, Viale Andrea Doria 6, 95125 Catania, Italy

${ }^{y}$ University Mohammed I, Laboratory of Physics of Matter and Radiations, B.P.717, Oujda 6000, Morocco

${ }^{z}$ Royal Netherlands Institute for Sea Research (NIOZ), Landsdiep 4,1797 SZ 't Horntje (Texel), The Netherlands $a{ }$ INFN - Sezione di Genova, Via Dodecaneso 33, 16146 Genova, Italy

$a b$ Dipartimento di Fisica dell'Università, Via Dodecaneso 33, 16146 Genova, Italy

${ }^{a c}$ Universiteit Utrecht, Faculteit Betawetenschappen, Princetonplein 5, 3584 CC Utrecht, The Netherlands

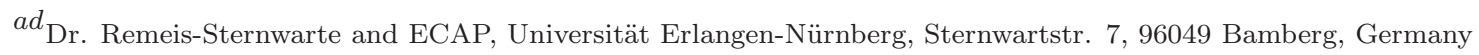

${ }^{a e}$ Moscow State University,Skobeltsyn Institute of Nuclear Physics,Leninskie gory, 119991 Moscow, Russia

af Dipartimento di Fisica ed Astronomia dell'Università, Viale Andrea Doria 6, 95125 Catania, Italy

$a g$ Direction des Sciences de la Matière - Institut de recherche sur les lois fondamentales de l'Univers - Service de Physique des Particules, CEA Saclay, 91191 Gif-sur-Yvette Cedex, France

$a h$ INFN -Sezione di Napoli, Via Cintia 80126 Napoli, Italy 
${ }^{a i}$ IPHC-Institut Pluridisciplinaire Hubert Curien - Université de Strasbourg et CNRS/IN2P3 23 rue du Loess, BP 28, 67037 Strasbourg Cedex 2, France

${ }^{a j}$ ITEP - Institute for Theoretical and Experimental Physics, B. Cheremushkinskaya 25, 117218 Moscow, Russia

$a k$ Dipartimento di Fisica dell'Università Federico II di Napoli, Via Cintia 80126, Napoli, Italy

Abstract. A search for high-energy neutrinos coming from the direction of the Galactic Centre is performed using the data recorded by the ANTARES neutrino telescope from 2007 to 2012. The event selection criteria are chosen to maximise the sensitivity to possible signals produced by the self-annihilation of weakly interacting massive particles accumulated around the centre of the Milky Way with respect to the atmospheric background. After data unblinding, the number of neutrinos observed in the line of sight of the Galactic Centre is found to be compatible with background expectations. The 90\% C.L. upper limits in terms of the neutrino+anti-neutrino flux, $\Phi_{\nu_{\mu}+\bar{\nu}_{\mu}}$, and the velocity averaged annihilation cross-section, $\left\langle\sigma_{\mathrm{Av}}\right\rangle$, are derived for the WIMP self-annihilation channels into $\mathrm{b} \overline{\mathrm{b}}, \mathrm{W}^{+} \mathrm{W}^{-}, \tau^{+} \tau^{-}, \mu^{+} \mu^{-}, \nu \bar{\nu}$. The ANTARES limits for $\left\langle\sigma_{\mathrm{A}} \mathrm{v}\right\rangle$ are shown to be the most stringent for a neutrino telescope over the WIMP masses $25 \mathrm{GeV}<\mathrm{M}_{\mathrm{WIMP}}<10 \mathrm{TeV}$.

Keywords: dark matter, neutrino telescope, indirect detection, Galactic Centre. 


\section{Contents}

1 Introduction 1

2 The ANTARES Neutrino Telescope 2

3 Signal and background simulation $\quad 4$

4 Optimisation of the event selection criteria $\quad 8$

5 Results and discussion $\quad 11$

6 Summary and conclusion $\quad 15$

\section{Introduction}

Observations in cosmology and astrophysics indicate that about $84 \%$ of the matter in the Universe, the so-called dark matter, is non-baryonic, non-relativistic, and does interact only through gravity. [1-4].

These observations involve the internal dynamics of galaxy clusters [5], the rotation curves of galaxies [6], weak lensing [7], also the Cosmic Microwave Background (CMB) from which the relic density of cold dark matter $(\mathrm{CDM})$ in the Universe is at present deduced to be $\Omega_{\mathrm{CDM}} \mathrm{h}^{2}=0.1199 \pm 0.0022$ (Planck+WMAP) $[8,9]$.

A common assumption is that dark matter is made of Weakly Interacting Massive Particles (WIMPs) that form halos in which the visible baryonic part of galaxies is embedded. There are a variety of candidates for WIMPs, among which those provided by theories based on supersymmetry (SUSY) attract a great deal of interest. In some classes of minimal supersymmetric (MSSM) and minimal universal extra-dimensional (mUED) extensions of the Standard Model (SM), the lightest particle (LP) is stable thanks to the conservation of a model-dependent parity that forbids its decay into SM particles. Consequently, these LPs can only annihilate in pairs, making them a possible WIMP candidate for dark matter [10, 11]. In these models, secondary high-energy neutrinos are produced from the decay of the LPs' self-annihilation products. In mUED models, neutrinos can even be produced directly in the self-annihilations, since there is no helicity suppression of fermion pair production.

The search for WIMPs can be performed either directly by recording the recoil energy of nuclei when WIMPs scatter off them in suitable detectors, or indirectly. The indirect approach, which is adopted here, exploits a radiation signature (gamma-ray, synchroton, positron, anti-proton or, as in this case, neutrino flux) produced by the self-annihilation of WIMPs accumulated in massive astrophysical objects such as the Galactic Centre (GC), the Sun or the Earth [12].

For the case of the GC, dealt with in this paper, where the density of dark matter in the galactic halo is supposed to be the highest, WIMPs self-annihilate to SM particles whose decay or hadronisation (if not directly to neutrinos) give rise to the production of high energy neutrinos which can travel from the GC to the Earth and be detected by neutrino telescopes.

In this paper, the indirect search for dark matter by looking for high-energy neutrinos coming from the GC, using the 2007-2012 data recorded by the ANTARES neutrino telescope, 
is described. The layout of the paper is as follows. In Section 2, the main features of the ANTARES neutrino telescope and the reconstruction algorithms used in this work are explained. In Section 3, the expected signal from WIMP self-annihilation from the GC, and the background expected from atmospheric muons and neutrinos are reported. In Section 4, the method used to optimise the selection of the neutrino events is described. Finally, the results obtained are discussed in Section 5, where limits on the neutrino plus anti-neutrino flux $\Phi_{\nu_{\mu}+\bar{\nu}_{\mu}}$ are derived from the absence of a signal. The corresponding 90\% C.L. upper limits on the velocity-averaged annihilation cross-section $\left\langle\sigma_{\mathrm{A}} \mathrm{v}\right\rangle$ are obtained for different benchmark channels of self-annihilation, and compared to the latest constraints from other experiments. In the following, neutrino will mean neutrino plus anti-neutrino, unless explicitly stated otherwise.

\section{The ANTARES Neutrino Telescope}

ANTARES is the first undersea neutrino telescope and the largest of its kind in the Northern Hemisphere [13]. It is located at $2475 \mathrm{~m}$ below the Mediterranean Sea level, $40 \mathrm{~km}$ offshore from Toulon (France) at $42^{\circ} 48^{\prime} \mathrm{N}$ and $6^{\circ} 10^{\prime} \mathrm{E}$. The telescope consists of 12 detection lines with 25 storeys each. A standard storey includes three optical modules (OMs) [14], each housing a 10-inch photomultiplier [15] and a local control module that contains the electronics $[16,17]$. The OMs are orientated $45^{\circ}$ downwards in order to optimise their acceptance to upgoing light and to avoid the effect of sedimentation and biofouling [18]. The length of a line is 450 $\mathrm{m}$ and the horizontal distance between neighbouring lines is $60-75 \mathrm{~m}$. In one of the lines, the upper storeys are dedicated to a test system for acoustic neutrino detection [19]. Similar acoustic devices are also installed in an additional line that contains instrumentation aimed to measure environmental parameters [20]. The location of the active components of the lines is known to better than $10 \mathrm{~cm}$ by a combination of tiltmeters and compasses in each storey and a series of acoustic transceivers (emitters and receivers) in certain storeys along the line and surrounding the telescope [21]. A common time reference is maintained in the full detector by means of a $25 \mathrm{MHz}$ clock signal broadcast from shore. The time offsets of the individual optical modules are determined in dedicated calibration facilities onshore and regularly monitored in situ by means of optical beacons distributed at various points of the apparatus which emit short light pulses through the water [22]. This allows a sub-nanosecond accuracy on the relative timing [23]. Additional information on the detector can be found in Ref. [13].

Data-taking started with the first 5 lines of the detector installed in 2007. The full detector was completed in May 2008 and has been operating continuously ever since, except for some short periods in which repair and maintenance operations have taken place. Other physics results using this data-taking period can be found elsewhere [24-32].

High-energy muon neutrinos interacting in the matter before the detector produce relativistic muons that can travel hundreds of metres and cross the detector or pass nearby. These muons induce Cherenkov light when travelling through the water, which is detected by the OMs. From the time and position information of the photons provided by the OMs, the direction of the muons, which is well correlated with that of the neutrinos, is reconstructed.

Two reconstruction algorithms are used in this paper. The first one is based on the minimisation of a $\chi^{2}$-like quality parameter of the reconstruction, $\mathrm{Q}$, which uses the difference between the expected and measured times of the detected photons, taking into account 


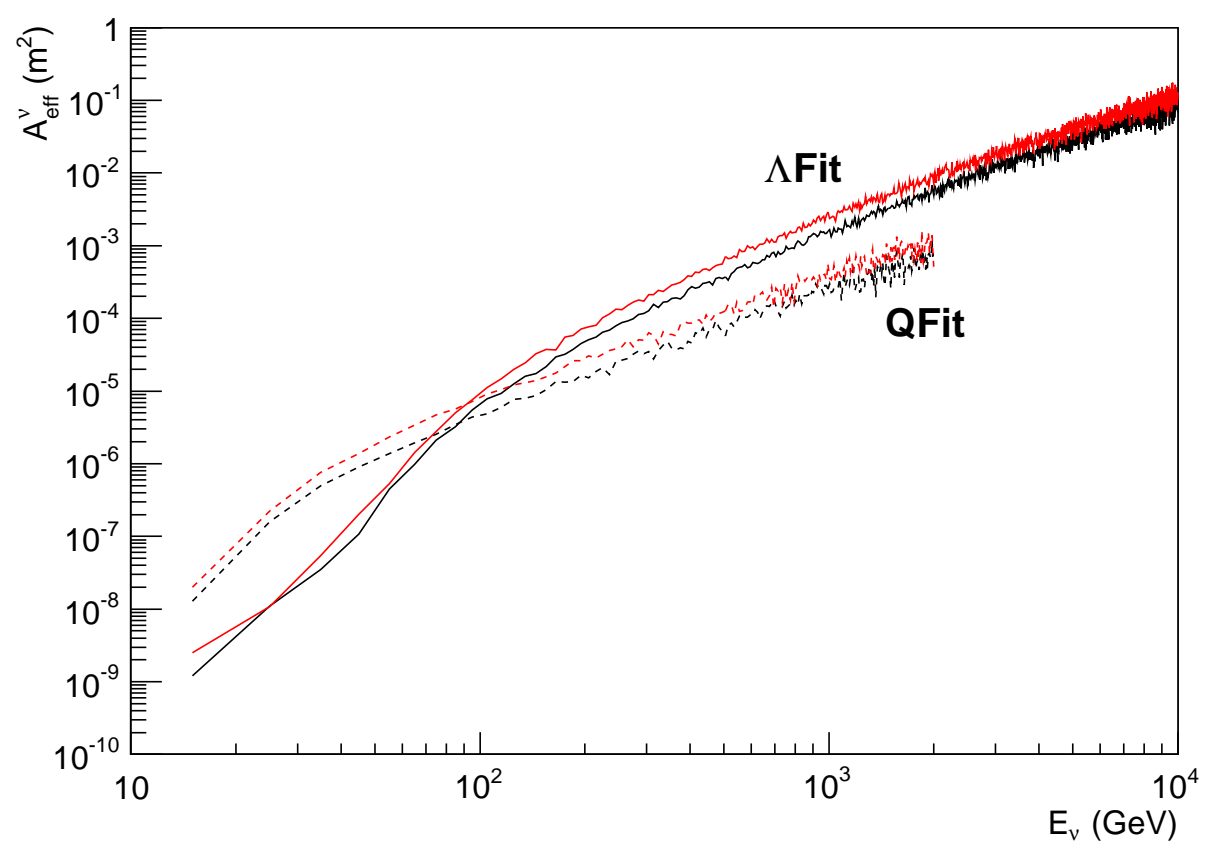

Figure 1. Effective area to muon neutrinos (black) and anti-neutrinos (red), $\mathrm{A}_{\text {eff }}^{\nu}\left(\mathrm{m}^{2}\right)$, for QFit (dashed line) and $\Lambda$ Fit (solid line), for the 12 line configuration of the detector. The QFit is limited here to its energy range of relevance, $\mathrm{E}_{\nu} \leq 2 \mathrm{TeV}$.

the effect of light absorption in the water [33]. The second algorithm consists of a multistep procedure to fit the direction of the muon track by maximising a likelihood ratio, $\Lambda$, which describes the quality of the reconstruction [27]. In addition to the $\Lambda$ parameter, the uncertainty of the muon track angle, $\beta$, is used for the track selection. These two algorithms are respectively called here QFit and $\Lambda$ Fit. QFit is used for muon events reconstructed in a single detection line (single-line events), and $\Lambda$ Fit for muon events reconstructed on more than one detection line (multi-line events) in order to reach the best efficiency of reconstruction in the entire neutrino energy range. For QFit and $\Lambda$ Fit, a selection on the quality parameter $\mathrm{Q}<0.8$ and the couple $\left(\Lambda>-5.7 ; \beta<0.5^{\circ}\right)$ have been used, respectively. These values of quality parameters are extracted from the optimisation step detailed in Section 4. QFit and $\Lambda$ Fit present different efficiencies of reconstruction, characterised by the effective areas for muon neutrinos, $A_{\text {eff }}^{\nu}\left(\mathrm{m}^{2}\right)$, which are shown in Figure 1 as a function of the energy of the primary neutrinos $\mathrm{E}_{\nu}(\mathrm{GeV})$. An effective area, $\mathrm{A}_{\text {eff }}^{\nu}$, at a given energy is defined as the ratio between the neutrino event rate $\left(\mathrm{s}^{-1}\right)$ in a detector and the neutrino flux $\left(\mathrm{m}^{-2} \cdot \mathrm{s}^{-1}\right)$ at that energy. As one can see, the QFit reconstruction strategy has a larger efficiency in the low energy regime with $\mathrm{A}_{\text {eff }}^{\nu}$ higher than the one obtained with $\Lambda$ Fit for $\mathrm{E}_{\nu}<100 \mathrm{GeV}$.

Beside the effective area, QFit and $\Lambda$ Fit yield different median angular resolutions, $\tilde{\alpha}=$ $\operatorname{med}\left[\left|\arccos \left(\tilde{\mathrm{d}}_{\text {rec }} \cdot \tilde{\mathrm{d}}_{\tilde{n}}\right)\right|\right]\left(^{\circ}\right)$ between the reconstructed muons, $\tilde{\mathrm{d}}_{\text {rec }}$, and the corresponding primary neutrinos, $\tilde{\mathrm{d}}_{\text {nu }}$, as shown in Figure 2. For QFit, since in single-line events only the zenith angle, $\theta$, is reconstructed, $\tilde{\alpha}$ is defined as the median of the difference between $\theta_{\text {rec }}$ and $\theta_{\text {nu }}, \tilde{\alpha}=\operatorname{med}\left[\left|\theta_{\text {rec }}-\theta_{\text {nu }}\right|\right]$. $\Lambda$ Fit yields a median angular resolution $6^{\circ}>\tilde{\alpha}>0.5^{\circ}$ for the primary neutrino energy range $100 \mathrm{GeV}<\mathrm{E}_{\nu}<10 \mathrm{TeV}$, whilst QFit reaches a $5.5^{\circ}>\tilde{\alpha}>3.8^{\circ}$ for $15 \mathrm{GeV}<\mathrm{E}_{\nu}<1 \mathrm{TeV}$. 


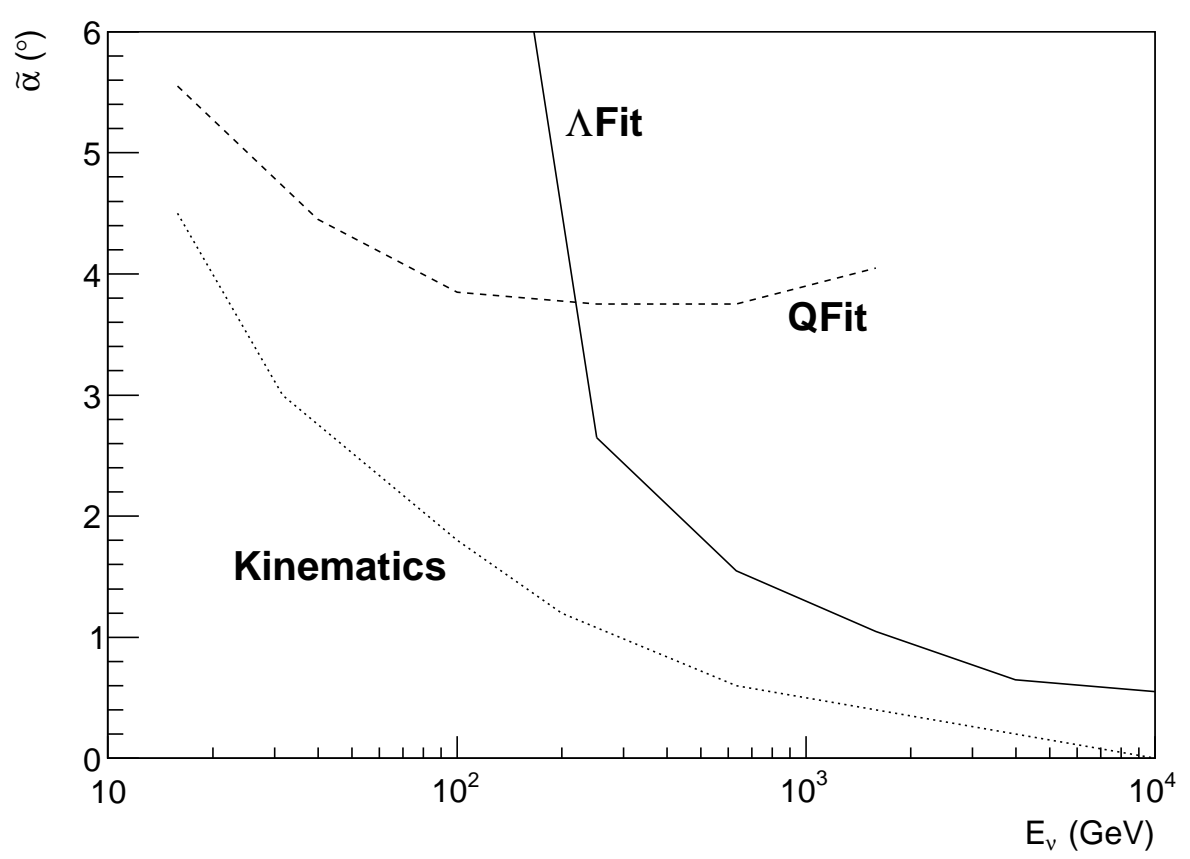

Figure 2. Median angular resolution, $\tilde{\alpha}\left(^{\circ}\right)$, on the muon neutrino direction, for the complete 12 line configuration of the detector. The performances for QFit (dashed line) and $\Lambda$ Fit (solid line) are illustrated, as is the kinematic counterpart (dotted line), the median angle between the primary neutrino and the outgoing muon at the vertex of interaction. For QFit, $\tilde{\alpha}$ is defined as the median resolution on the zenith angles $\theta_{\text {rec,nu }}$ (see text for details).

Both reconstruction algorithms are used in the search and the one with the best performance in a given energy range is selected to obtain the best possible limits in that range.

\section{Signal and background simulation}

The energy spectrum of muon neutrinos arriving at the Earth's surface from WIMP selfannihilation occurring in the GC's vicinity is computed using Ref. [34]. The muon neutrinos resulting from the self-annihilation channels in SM particles are propagated to the Earth for 17 different WIMP masses in the range from $25 \mathrm{GeV}$ to $10 \mathrm{TeV}$. To compute the initial energy spectra at the GC, five benchmark self-annihilation channels of WIMP-like dark matter particles into SM particles are used:

$$
\text { WIMP WIMP } \rightarrow \mathrm{b} \overline{\mathrm{b}}, \mathrm{W}^{+} \mathrm{W}^{-}, \tau^{+} \tau^{-}, \mu^{+} \mu^{-}, \nu_{\alpha} \bar{\nu}_{\alpha} .
$$

Also, neutrinos flavours, $\alpha=\mathrm{e}, \mu, \tau$, can be produced directly or as subsequent decay products of the SM particles listed above. The propagation of the neutrinos includes the three-flavour neutrino oscillations in vacuum along the line of sight from the GC to the surface of the Earth using the values from Table 8 in Ref. [35]. The muon neutrino energy spectrum can be written as:

$$
\left.\frac{\mathrm{dN}_{\nu_{\mu}}}{\mathrm{dE}_{\nu_{\mu}}}\right|_{\oplus}=\left.\sum_{\alpha} \mathrm{P}\left(\nu_{\alpha} \rightarrow \nu_{\mu}\right) \frac{\mathrm{dN}_{\nu_{\alpha}}}{\mathrm{dE}_{\nu_{\alpha}}}\right|_{\ominus},
$$




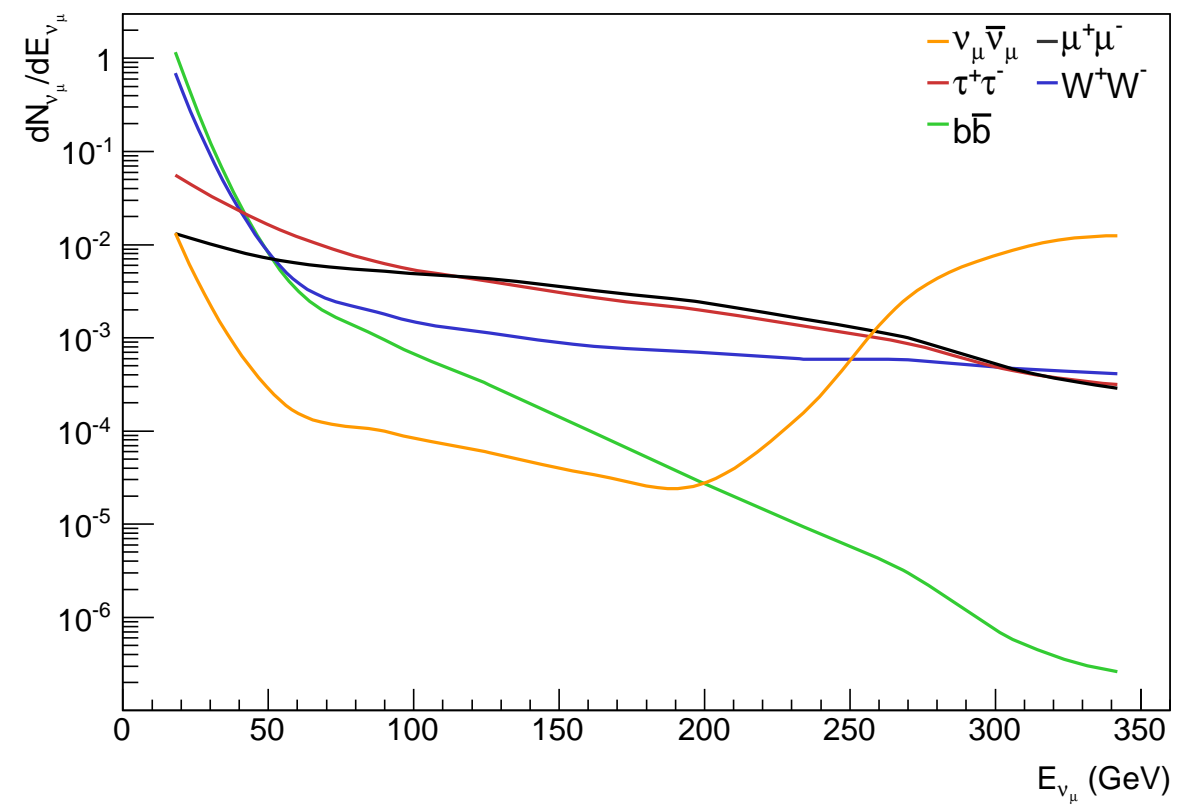

Figure 3. Example of muon neutrino energy spectra at the surface of the Earth $\mathrm{dN}_{\nu_{\mu}} /\left.\mathrm{dE}_{\nu_{\mu}}\right|_{\oplus}$ as a function of their energy $\mathrm{E}_{\nu_{\mu}}$ for a WIMP mass $\mathrm{M}_{\mathrm{WIMP}}=360 \mathrm{GeV}$. The primary self-annihilation channels: WIMP WIMP $\rightarrow$ b $\bar{b}$ (green), $\mathrm{W}^{+} \mathrm{W}^{-}$(blue), $\tau^{+} \tau^{-}$(red), $\mu^{+} \mu^{-}$(black), $\nu_{\mu} \bar{\nu}_{\mu}$ (orange) are shown.

where $\mathrm{dN}_{\nu_{\mu}} /\left.\mathrm{dE}_{\nu_{\mu}}\right|_{\oplus}$ is the muon neutrino energy spectrum at the Earth, $\mathrm{P}\left(\nu_{\alpha} \rightarrow \nu_{\mu}\right)$ is the probability to observe the oscillation $\nu_{\alpha} \rightarrow \nu_{\mu}$, where $\alpha=\mathrm{e}, \mu, \tau$, and $\mathrm{dN}_{\nu_{\alpha}} /\left.\mathrm{dE}_{\nu_{\alpha}}\right|_{\ominus}$ is the corresponding energy spectrum at the GC. Examples of muon neutrino energy spectra at the surface of the Earth, $\mathrm{dN}_{\nu_{\mu}} /\left.\mathrm{dE}_{\nu_{\mu}}\right|_{\oplus}$, are shown in Figure 3 as a function of their energy $\mathrm{E}_{\nu_{\mu}}(\mathrm{GeV})$ (the spectra in muon anti-neutrinos are identical), for an indicative mass $\mathrm{M}_{\text {WIMP }}=360 \mathrm{GeV}$. In this figure, the three-flavour oscillations process in vacuum over the GC-Earth line of sight is used as expressed in Equation 3.2. As with the dark matter search in the direction of the Sun [30], WIMP WIMP $\rightarrow \mathrm{b} \bar{b}$ is the softest channel of muon neutrino production. The hardest channel of muon neutrino production from the GC is the primary WIMP WIMP $\rightarrow \nu_{\alpha} \bar{\nu}_{\alpha}$. It is calculated taking into account the contribution of electroweak corrections discussed in Ref. [34]. The three other channels, WIMP WIMP $\rightarrow$ $\mathrm{W}^{+} \mathrm{W}^{-}, \tau^{+} \tau^{-}, \mu^{+} \mu^{-}$, present a hard contribution to the full spectrum of muon neutrinos at Earth. It can be noted that the channels to $\tau^{+} \tau^{-}$and $\mu^{+} \mu^{-}$differ only for $\mathrm{E}_{\nu_{\mu}}<100 \mathrm{GeV}$ in this example.

In order to be as model-independent as possible, a self-annihilation branching ratio $\mathrm{BR}=1$ is used for each of the channels in Expression 3.1. Beyond-the-SM particle physics with $\mathrm{BR}<1$ can be accommodated by scaling the fluxes in Equation 3.2 linearly with the appropriate branching ratio.

The main backgrounds for this analysis are atmospheric muons and neutrinos, both produced in the interactions of cosmic rays with the Earth's atmosphere. Downgoing atmo- 

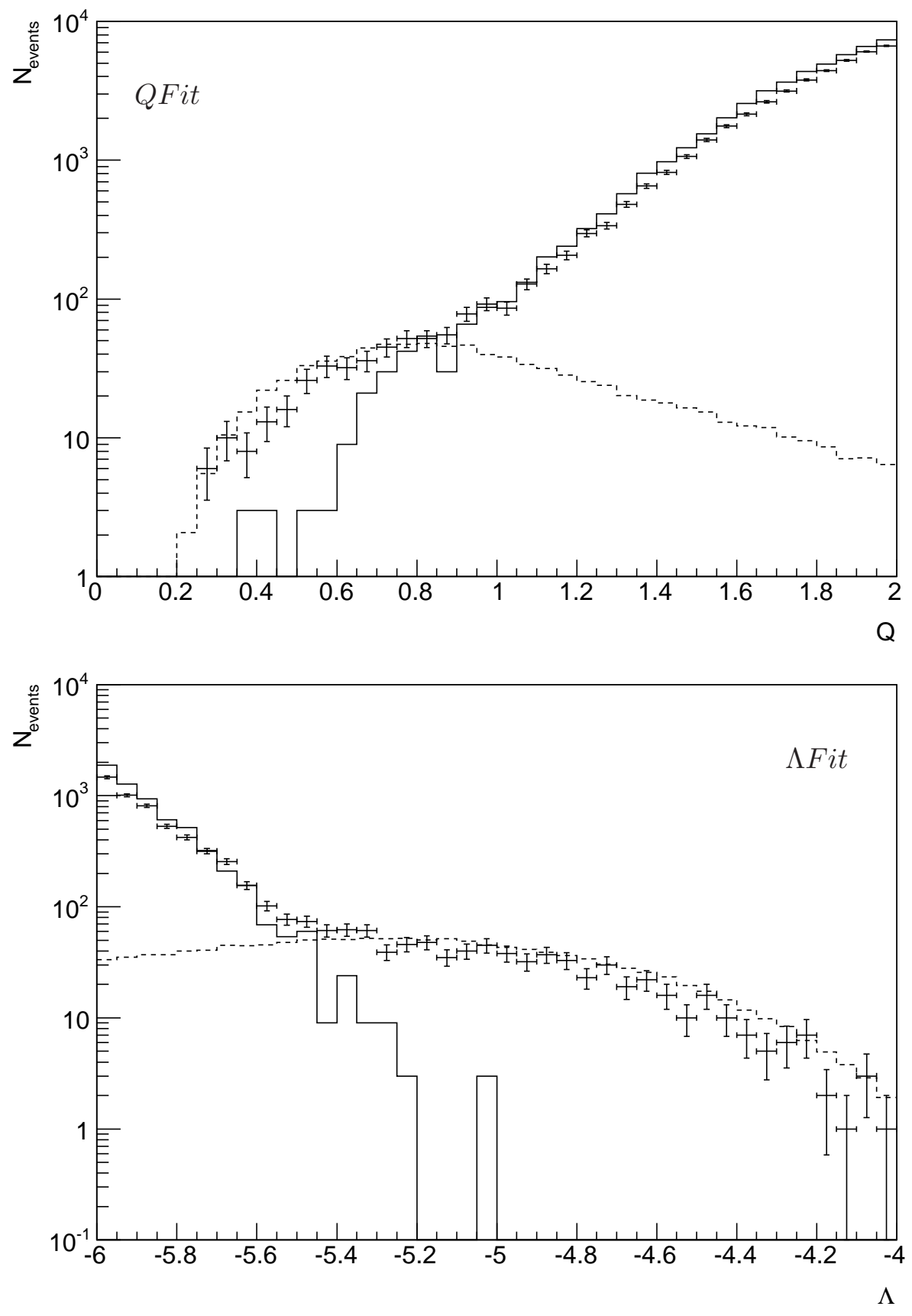

Figure 4. Distribution of the number of reconstructed events, $N_{\text {events }}$, for the QFit (top) and $\Lambda$ Fit (bottom) algorithms as a function of their respective track reconstruction parameter, $\mathrm{Q}$ and $\Lambda$. The expectations according to simulations for atmospheric neutrinos (dashed line), atmospheric muons (solid line), and the data (black crosses) for 2012 are presented. For QFit, only the upgoing events, $\cos \left(\theta_{\text {rec }}\right)>0$, are used. For $\Lambda$ Fit, the uncertainty of the muon track direction angle is required to be $\beta<0.5$, and the field of view is extended to $\cos \left(\theta_{\text {rec }}\right)>-0.1$.

spheric muons dominate the trigger rate, which ranges from 3 to $10 \mathrm{~Hz}$ depending on the trigger conditions. They are simulated using MUPAGE [36]. Upgoing atmospheric neutrinos, 

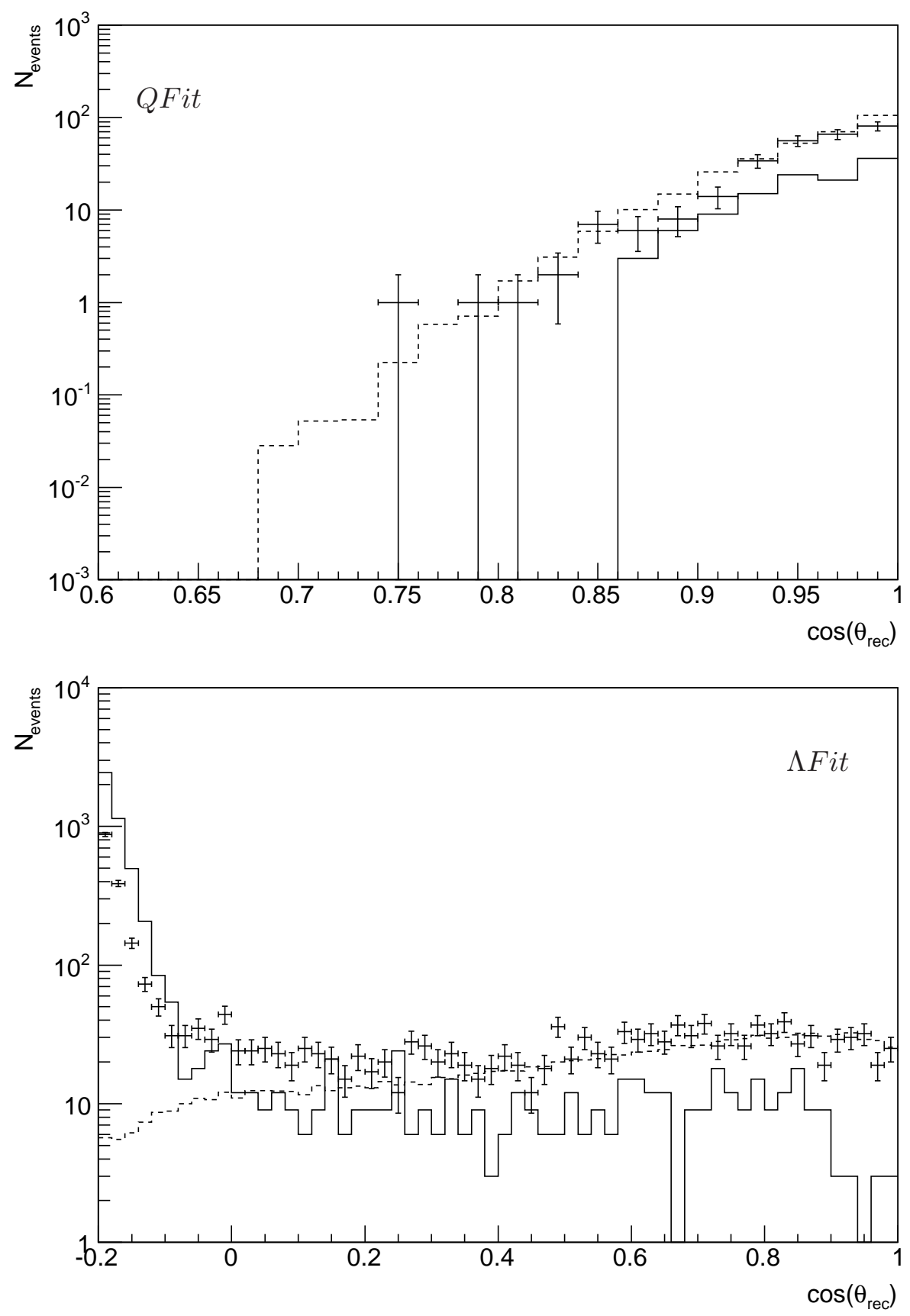

Figure 5. Distribution of the number of reconstructed events, $N_{\text {events }}$, for the QFit (top) and $\Lambda$ Fit (bottom) algorithms as a function of the cosine of their zenith angle $\cos \left(\theta_{\text {rec }}\right)$. The expectations according to simulations for atmospheric neutrinos (dashed line), atmospheric muons (solid line), and the data (black crosses) for 2012 are shown. For QFit and $\Lambda$ Fit, the cuts $\mathrm{Q}<0.8$ and $(\Lambda>-5.7 ; \beta<$ $0.5)$ are used, respectively.

which are recorded at a rate of $\sim 50 \mu \mathrm{Hz}$ (about four per day), are simulated according to the parameterisation of the atmospheric $\nu_{\mu}$ flux from Ref. [37] in the energy range from $10 \mathrm{GeV}$ to $10 \mathrm{PeV}$ using GENHEN [38]. Furthermore, the propagation of muon tracks is simulated 
with the KM3 package [38]. The Cherenkov light produced in the vicinity of the detector is propagated taking into account light absorption and scattering in sea water [39]. The characteristics of the PMTs are taken from Ref. [14] and the overall geometry corresponds to the different layouts of the ANTARES detector during each data-taking period.

The simulated effective area is used to evaluate the expected signal from WIMP selfannihilations. The expected background is estimated from the scrambled data in order to avoid systematic uncertainties from the simulation. The scrambling consists in a uniform randomisation of the UTC time of the events in the data-taking period. The zenith and azimuth angles of the reconstructed tracks are kept so as to preserve the angular response of the detector in the optimisation of the selection criteria. This procedure provides a means to follow a data blinding strategy while using all the relevant information on the detector performance.

The criteria to select events and to reduce the background from atmospheric muons and neutrinos, and to improve the sensitivity of ANTARES to a dark matter signal, are devised following a blind procedure on the srambled data before performing the analysis on the data.

\section{Optimisation of the event selection criteria}

The data used in this search were recorded between the $27^{\text {th }}$ of January 2007 and the $31^{\text {st }}$ of October 2012, corresponding to a total livetime of about 1321 days. This livetime is not corrected for the visibility of the GC. During this time, the detector consisted of 5 lines for most of 2007 and 12 lines from 2008 to 2012, with short periods of 8,9 and 10 lines.

The QFit and $\Lambda$ Fit methods, as introduced in Section 2, are respectively used for singleand multi-line track fit reconstructions. Pre-selection cuts are applied to obtain an event sample, dominated by well-reconstructed atmospheric neutrinos. For QFit, only upgoing events are used, i.e. $\cos \left(\theta_{\text {rec }}\right)>0$. For $\Lambda$ Fit, the error estimate of the reconstructed muon track direction, $\beta$, should be smaller than 0.5 , and a cut $\cos \left(\theta_{\text {rec }}\right)>-0.1$ is applied. The distributions of the track fit quality parameters, $\mathrm{Q}$ and $\Lambda$, for the resulting event samples are shown in Figure 4 for both reconstruction methods. By choosing additionally the cuts $\mathrm{Q}<0.8$ and $\Lambda>-5.7$, a purity of $69 \%$ and $72 \%$ in muon neutrinos is reached for QFit and $\Lambda$ Fit, respectively. The zenith angle distributions of the event samples after the cuts in $\mathrm{Q}$ and $\Lambda$ are shown in Figure 5. Single-line events, as reconstructed by QFit, are mostly found close to the vertical direction, as illustrated in Figure 5 (top).

After the pre-selection, the angular separation, $\Psi$, between the reconstructed track and the GC's direction is used in an optimisation of the model rejection factor [40] as a function of the WIMP mass, $\mathrm{M}_{\text {WIMP }}$. For each WIMP mass and annihilation channels listed in the Tables $1+2+3+4$, the selected value $\Psi$ is the one that minimises the average $90 \%$ confidence level (C.L.) upper limit on the $\nu_{\mu}+\bar{\nu}_{\mu}$ flux, $\bar{\Phi}_{\nu_{\mu}+\bar{\nu}_{\mu}}$, defined as:

$$
\bar{\Phi}_{\nu_{\mu}+\bar{\nu}_{\mu}}=\frac{\bar{\mu}^{90 \%}}{\sum_{\mathrm{i}} \overline{\mathrm{A}}_{\text {eff }}^{\mathrm{i}}\left(\mathrm{M}_{\mathrm{WIMP}}\right) \times \mathrm{T}_{\mathrm{eff}}^{\mathrm{i}}},
$$

where the index i denotes the periods with different detector configurations, $\bar{\mu}^{90 \%}$ is the average upper limit of the background from scrambled data at 90\% C.L. (computed using a Poisson distribution in the Feldman-Cousins approach [41]), and $\mathrm{T}_{\text {eff }}^{\mathrm{i}}$ is the effective livetime for each detector configuration. The effective area averaged over the neutrino energy, $\overline{\mathrm{A}}_{\text {eff }}^{\mathrm{i}}\left(\mathrm{M}_{\text {WIMP }}\right)$, is defined as: 


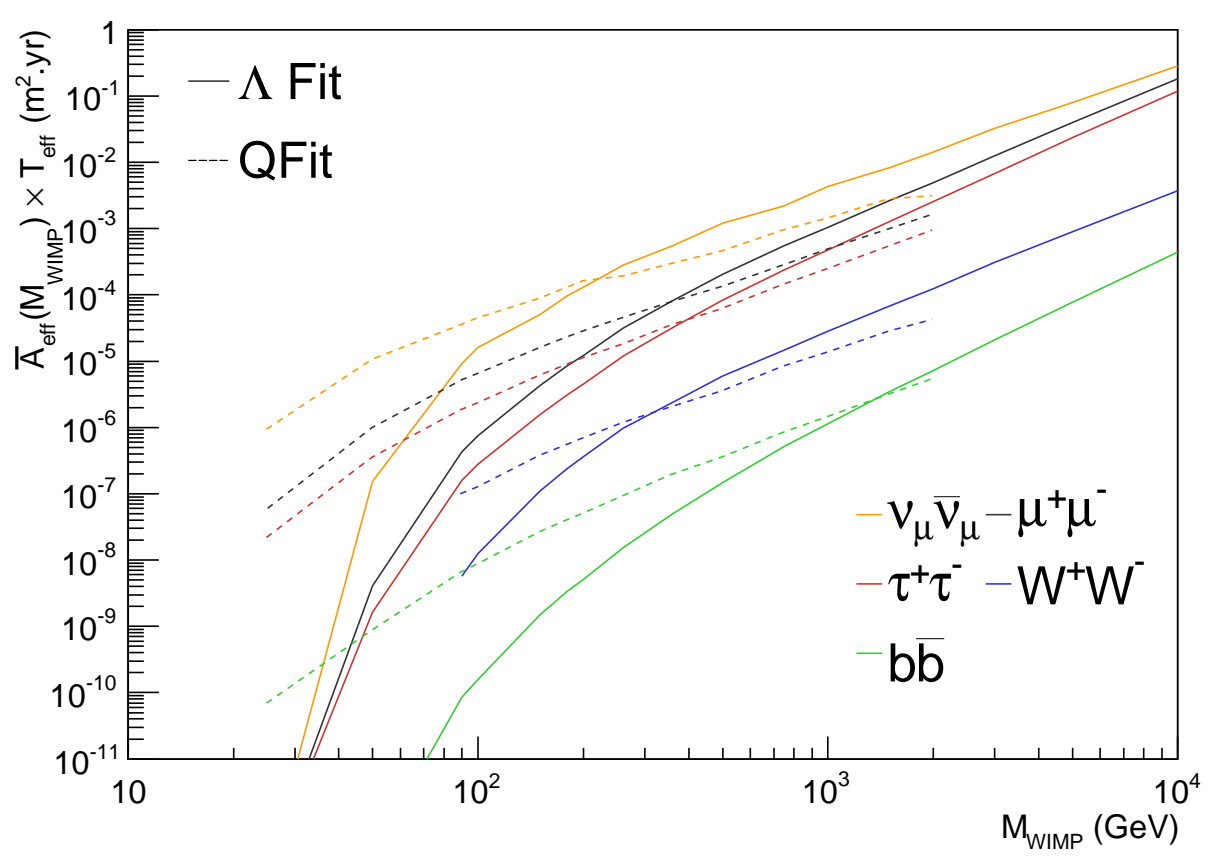

Figure 6. Example of the acceptance $\overline{\mathrm{A}}_{\mathrm{eff}}\left(\mathrm{M}_{\mathrm{WIMP}}\right) \times \mathrm{T}_{\mathrm{eff}}\left(\mathrm{m}^{2} \cdot \mathrm{yr}\right)$ to the signal of WIMP self-annihilation towards the GC as a function of WIMP masses $25 \mathrm{GeV}<\mathrm{M}_{\text {WIMP }}<10 \mathrm{TeV}$. The acceptances for the primary self-annihilation channels (from bottom to top) WIMP WIMP $\rightarrow$ $\mathrm{b} \overline{\mathrm{b}}$ (green), $\mathrm{W}^{+} \mathrm{W}^{-}$(blue), $\tau^{+} \tau^{-}$(red), $\mu^{+} \mu^{-}$(black), $\nu_{\mu} \bar{\nu}_{\mu}$ (orange) are shown. QFit (dashed lines) and $\Lambda$ Fit (solid lines) are compared.

$$
\overline{\mathrm{A}}_{\text {eff }}^{\mathrm{i}}\left(\mathrm{M}_{\mathrm{WIMP}}\right)=\frac{\left.\int_{\mathrm{E}_{\nu_{\mu}}^{\mathrm{th}}}^{\mathrm{M}_{\mathrm{WIMP}}}\left(\mathrm{A}_{\text {eff }}^{\nu_{\mu}, \mathrm{i}}+\mathrm{A}_{\text {eff }}^{\bar{\nu}_{\mu}, \mathrm{i}}\right) \frac{\mathrm{dN}_{\nu_{\mu}}}{\mathrm{dE}_{\nu_{\mu}}}\right|_{\oplus} \mathrm{dE_{ \nu _{ \mu } }}}{2 \int_{0}^{\mathrm{M}_{\mathrm{WIMP}}} \frac{\mathrm{dN}_{\nu_{\mu}}}{\mathrm{dE}_{\nu_{\mu}}} \mathrm{dE}_{\nu_{\mu}}},
$$

where $\mathrm{E}_{\nu_{\mu}}^{\mathrm{th}} \simeq 15 \mathrm{GeV}$ is the energy threshold for neutrino detection in ANTARES, $\mathrm{dN}_{\nu_{\mu}} /\left.\mathrm{dE}_{\nu_{\mu}}\right|_{\oplus}=$ $\mathrm{dN}_{\bar{\nu}_{\mu}} /\left.\mathrm{dE}_{\bar{\nu}_{\mu}}\right|_{\oplus}$ is the energy spectrum of the neutrinos at the surface of the Earth as shown in Figure 3, and $\mathrm{A}_{\text {eff }}^{\nu_{\mu} / \bar{\nu}_{\mu}}$ is the effective area of ANTARES as a function of the neutrino or antineutrino energy for tracks coming from the direction of the GC (Figure 1 for illustration). Due to their different cross-sections, the effective areas for neutrinos and anti-neutrinos are slightly different and therefore are considered separately, whereas the fluxes of muon neutrinos and anti-neutrinos from the GC are identical.

The optimisation procedure provides a set of optimum values of angular separation to the GC, $\Psi$, for each mass of the WIMP and for each benchmark channel. The distributions of $\Psi$ as a function of the WIMP mass, $\mathrm{M}_{\mathrm{WIMP}}$, are given in Tables $1+2$ and $3+4$. Regardless of self-annihilation channels and reconstruction algorithms, an optimised angular separation $\Psi$ to the GC is wider for low M WIMP due to the degradation of the angular resolution $\tilde{\alpha}$ at low neutrino energy (Figure 2). Moreover, the optimimum $\Psi$ for a given $\mathrm{M}_{\text {WIMP }}$ depends on the softness of the self-annihilation channel, $\Psi$ being wider the softer the channel. 


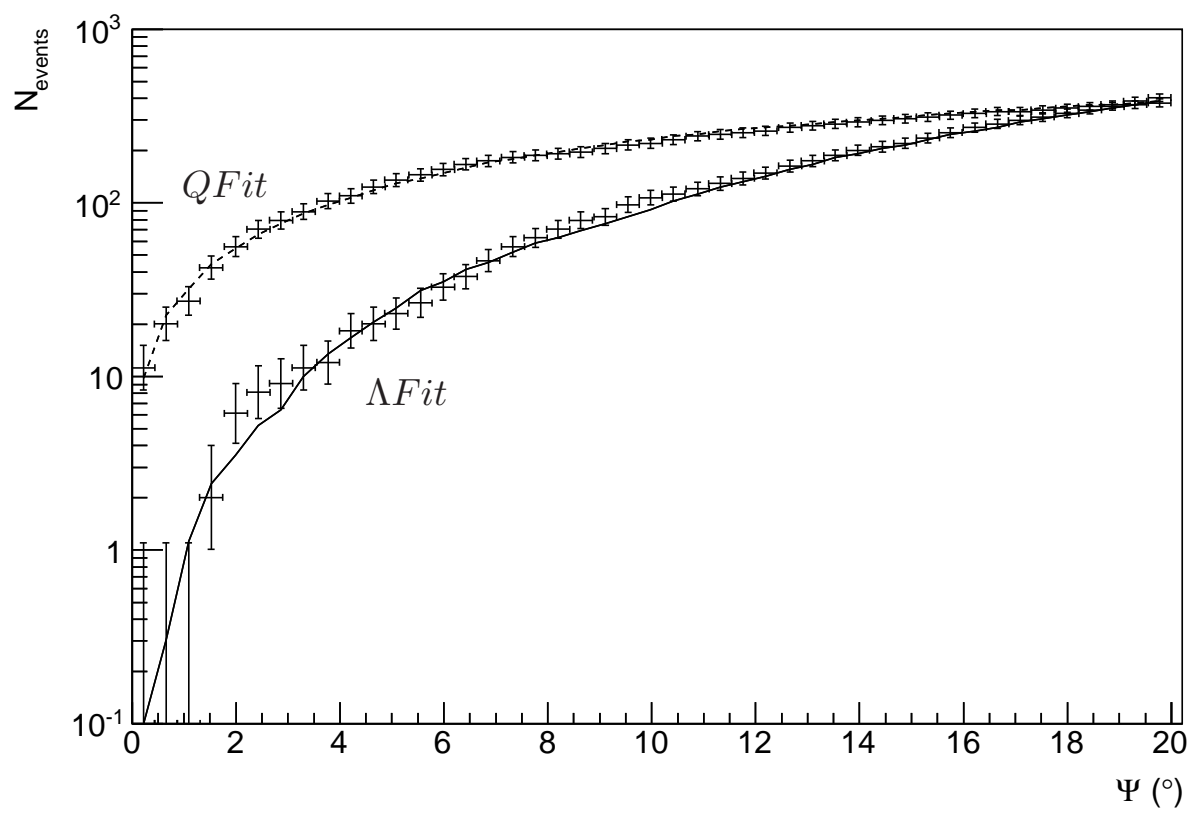

Figure 7. Distribution of the number of events as a function of the angular separation, $\Psi$, in the direction of the GC for the expected backgrounds (dashed line for QFit, solid line for $\Lambda$ Fit) compared to the data (black crosses) from QFit and $\Lambda$ Fit. A $1 \sigma$ Gaussian uncertainty is shown for each data point.

An example of the acceptance $\overline{\mathrm{A}}_{\text {eff }}\left(\mathrm{M}_{\mathrm{WIMP}}\right) \times \mathrm{T}_{\text {eff }}$ is shown in Figure 6 . The visibility of the GC and the data-taking periods from 2007 to 2012 are included. The QFit (dashed lines) and $\Lambda$ Fit (solid lines) are compared for each self-annihilation channel, inside their respective angular separations, $\Psi=10^{\circ}$ and $\Psi=2^{\circ}$, that are typical over all the WIMP masses (Tables $1+2+3+4$ ). Notice that the softer a self-annihilation channel is, the lower the corresponding acceptance, due to the decrease of the effective area $\mathrm{A}_{\text {eff }}^{\nu}$ shown in Figure 1 towards the low neutrino energies. Also, the channels WIMP WIMP $\rightarrow \nu_{\alpha} \bar{\nu}_{\alpha}$ and WIMP WIMP $\rightarrow$ b $\bar{b}$ show respectively the largest and smallest acceptance to a WIMP selfannihilation signal. This will respectively induce the best and worse limits for a neutrino flux $\Phi_{\nu_{\mu}+\bar{\nu}_{\mu}}$, as shown in Equation 4.1. The $\overline{\mathrm{A}}_{\mathrm{eff}}\left(\mathrm{M}_{\mathrm{WIMP}}\right) \times \mathrm{T}_{\mathrm{eff}}$ distribution of the $W^{+} W^{-}$ channel is kinematically allowed for $M_{\text {WIMP }}>M_{W}=80.4 \mathrm{GeV}$ [4]. However, the low mass region, $25 \mathrm{GeV}<\mathrm{M}_{\mathrm{WIMP}}<\mathrm{M}_{\mathrm{W}}$, is probed by other channels. Moreover, the energy range for which QFit is more efficient than $\Lambda$ Fit will change slightly according to the softness of the self-annihilation channel. As can be seen in Figure 3, most of the contribution of the $b \bar{b}$ channel lies in the range $\mathrm{E}_{\nu_{\mu}}<100 \mathrm{GeV}$, for $\mathrm{M}_{\text {WIMP }}=360 \mathrm{GeV}$, where QFit yields a higher effective area compared to $\Lambda$ Fit. Therefore, QFit is preferred over the range of masses $\mathrm{M}_{\text {WIMP }}<1.5 \mathrm{TeV}$ to compute the best $b \bar{b}$ limit. Furthermore, this evolves according to the self-annihilation channel spectrum and the WIMP mass, as described in the following Section 5 . 


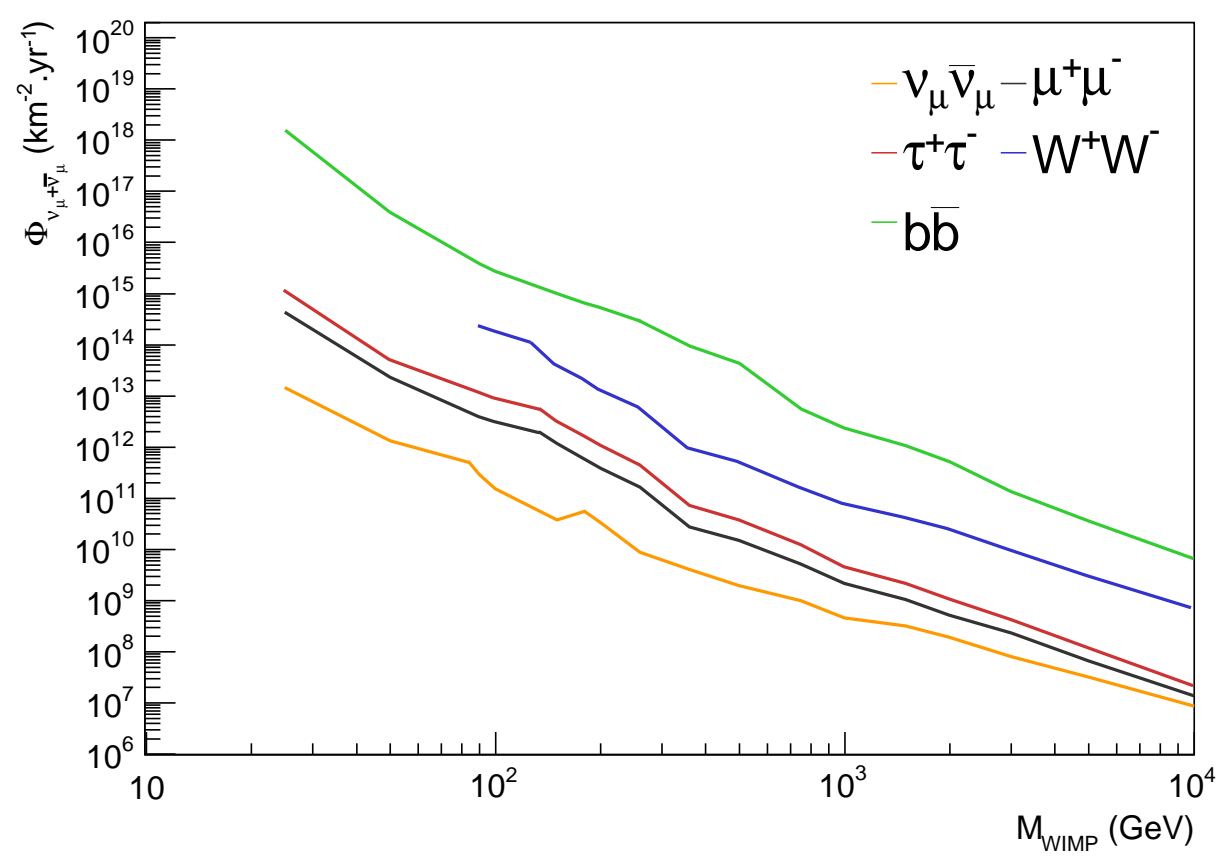

Figure 8. $90 \%$ C.L. upper limits on the neutrino flux, $\Phi_{\nu_{\mu}+\bar{\nu}_{\mu}}$, as a function of the WIMP mass in the range $25 \mathrm{GeV}<\mathrm{M}_{\mathrm{WIMP}}<10 \mathrm{TeV}$ for the self-annihilation channels (from top to bottom) WIMP WIMP $\rightarrow$ b $\bar{b}$ (green), $\mathrm{W}^{+} \mathrm{W}^{-}$(blue), $\tau^{+} \tau^{-}$(red), $\mu^{+} \mu^{-}$(black), $\nu_{\mu} \bar{\nu}_{\mu}$ (orange). The QFit and $\Lambda$ Fit results are combined.

\section{Results and discussion}

Given the pre-selection and optimisation processes described in Section 2 and 4, a total of 369 and 401 events for the unblinded 2007-2012 data are found within an angular separation $\Psi$ of $20^{\circ}$ from QFit and $\Lambda$ Fit, respectively. This angle is large enough to contain all values of the optimal angular separation $\Psi$ for all WIMP masses and for each channel. Figure 7 shows the integrated distribution of the reconstructed event numbers as a function of their relative angular separation to the GC, $\Psi$. Data are in good agreement with the expected background for both reconstruction algorithms. As no statistically significant excess is observed in the direction of the GC, upper limits on a neutrino flux can be set.

The $90 \%$ C.L. upper limits on the $\nu_{\mu}+\bar{\nu}_{\mu}$ flux at Earth, $\Phi_{\nu_{\mu}+\bar{\nu}_{\mu}}$, are computed from the data according to Equation 4.1, where the average 90\% C.L. upper limit $\bar{\mu}^{90 \%}$ is replaced by the upper limit at $90 \%$ C.L. on the number of observed events, $\mu^{90 \%}$. Systematic uncertainties are taken into account and included in the evaluation of the limits using the Pole software following the approach described in Ref. [52]. The total systematic uncertainty on the detector efficiency is about $20 \%$ and comes mainly from the uncertainties on the average quantum efficiency and the angular acceptance of the PMTs, and the sea water absorption length. The detailed uncertainty study is described in Ref. [23]. This total systematic uncertainty translates into a degradation of the upper limits between $3 \%$ and $6 \%$, depending on the WIMP mass. The corresponding limits are presented in Figure 8 for all the benchmark self-annihilation channels. In this figure, the results from QFit and $\Lambda$ Fit are combined to reach the best upper limit over the whole $\mathrm{M}_{\text {WIMP }}$ range. Given its soft energy spectrum 


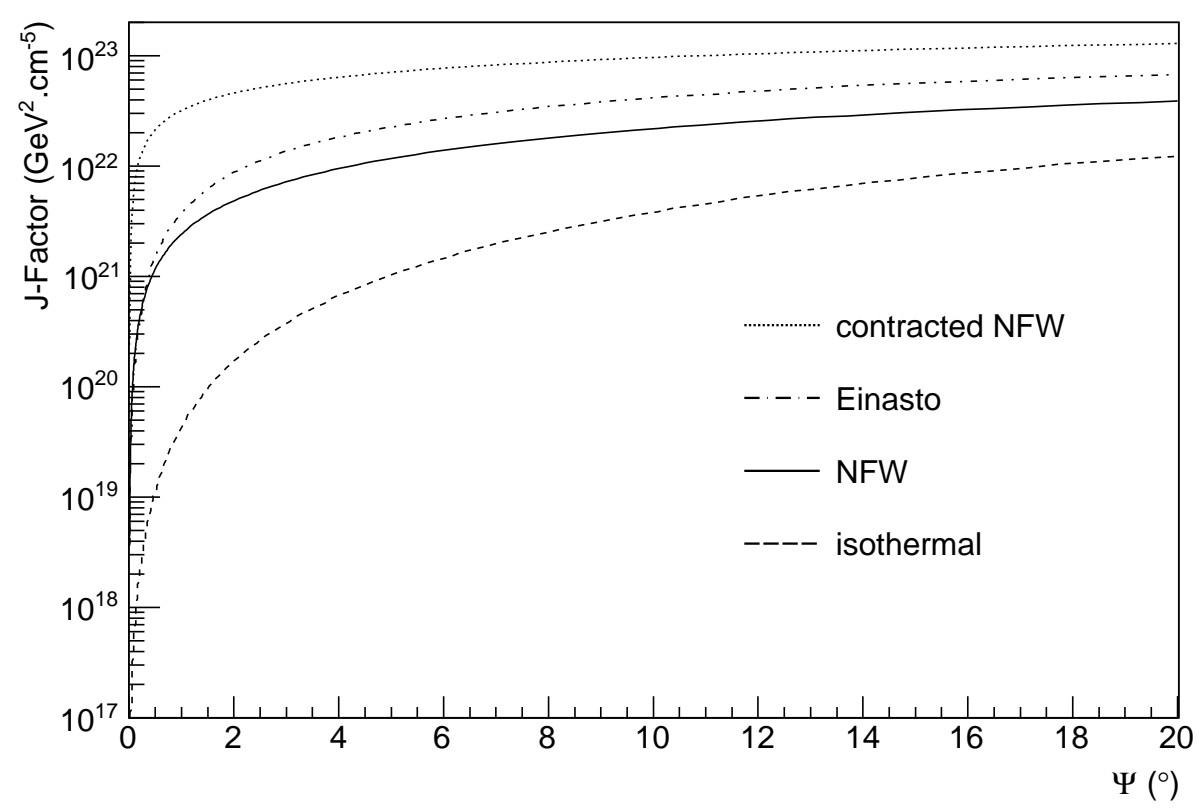

Figure 9. Distributions of the integrated J-Factor $\left(\mathrm{GeV}^{2} \cdot \mathrm{cm}^{-5}\right)$ as a function of the angular separation to the GC, $\Psi$. The dark matter density profiles: a contracted NFW [55] (dotted line), Einasto [53] (dot-dashed line), NFW [42] (solid line) and isothermal [54] (dashed line), are compared.

(Figure 6), the channel $b \bar{b}$ yields the least stringent limit, while it is the opposite for $\nu_{\alpha} \bar{\nu}_{\alpha}$. The upper limits, $\Phi_{\nu_{\mu}+\bar{\nu}_{\mu}}$, improve from low to high WIMP masses, as expected from the general hardness of the neutrino spectra at the surface of the Earth, the absence of interaction during the neutrino propagation along the line of sight from the GC (no absorption or diffusion are present), and the tightness of the optimum angular separation around the GC, $\Psi$, for high WIMP masses (Table 4).

The neutrino flux from the self-annihilation of dark matter particles at the GC can be expressed as:

$$
\begin{aligned}
& \Phi_{\nu_{\mu}+\bar{\nu}_{\mu}}=\Xi_{\nu_{\mu}+\bar{\nu}_{\mu}}^{\mathrm{PP}} \times \mathrm{J}(\Delta \Omega), \\
& \Xi_{\nu_{\mu}+\bar{\nu}_{\mu}}^{\mathrm{PP}}=\frac{1}{4 \pi} \frac{<\sigma_{\mathrm{A}} \mathrm{V}>}{2 \mathrm{M}_{\mathrm{WIMP}}^{2}} \mathrm{~N}_{\nu_{\mu}+\bar{\nu}_{\mu}}, \\
& \mathrm{J}(\Delta \Omega)=\int_{\Delta \Omega} \int \rho_{\mathrm{DM}}^{2} \mathrm{dl} \mathrm{d} \Omega,
\end{aligned}
$$

with $\Xi_{\nu_{\mu}+\bar{\nu}_{\mu}}^{\mathrm{PP}}$ as the particle physics term, which depends on the WIMP velocity averaged self-annihilation cross-section, $\left\langle\sigma_{\mathrm{AV}}\right\rangle$, and the number of neutrinos, $N_{\nu_{\mu}+\bar{\nu}_{\mu}}$, that reach the surface of the Earth. $N_{\nu_{\mu}+\bar{\nu}_{\mu}}$ is computed from the integration over the neutrino energy of the GC's self-annihilation spectra. The astrophysical J-Factor is the integral over the line of sight, $l$, and the solid angle around the GC, $\Omega$, of the dark matter density, $\rho_{\mathrm{DM}}$, squared. The density $\rho_{\text {DM }}$ depends on the chosen dark matter galactic halo profile. The NavarroFrenk-White (NFW) profile [42] is selected as a reference for which the density profile of dark matter is expressed as: 


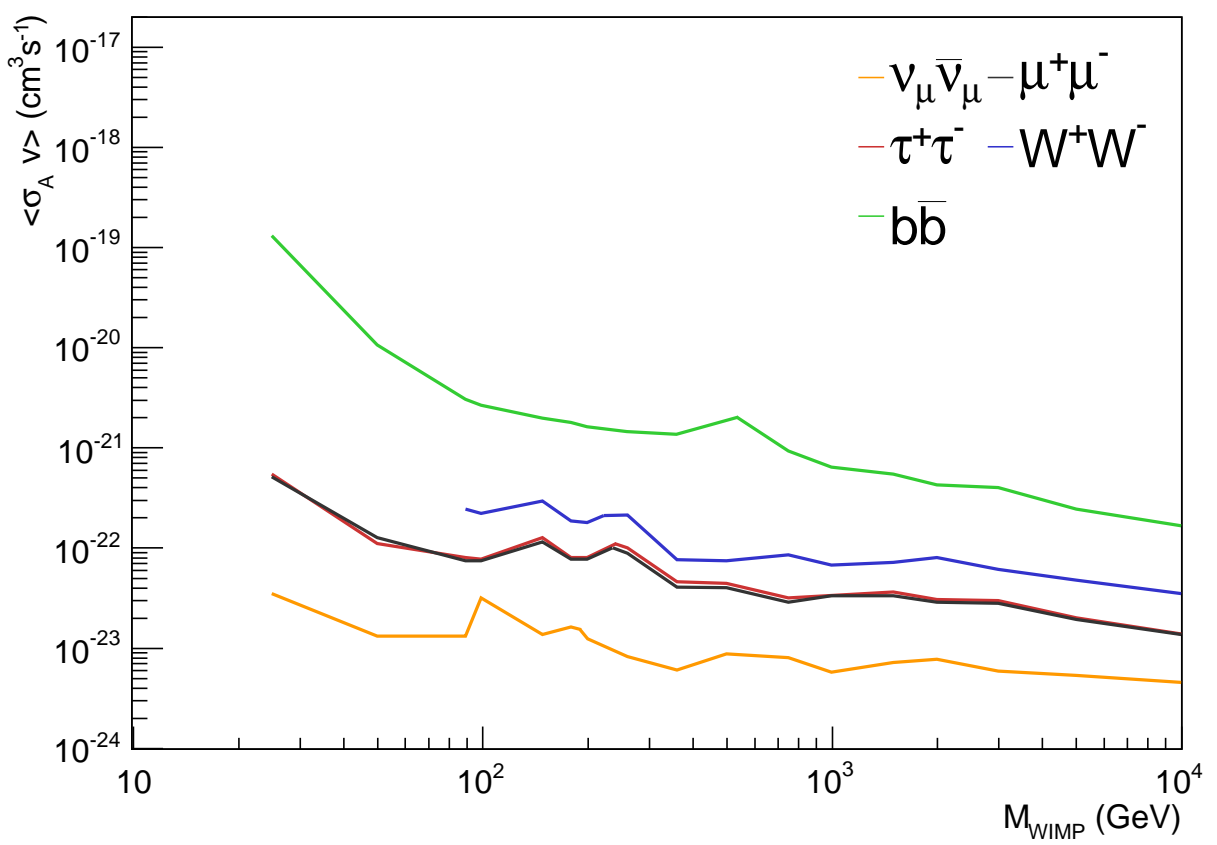

Figure 10. The 90\% C.L. upper limits on the WIMP velocity averaged self-annihilation crosssection, $\left\langle\sigma_{\mathrm{AV}}>\right.$, as a function of the WIMP mass in the range $25 \mathrm{GeV}<\mathrm{M}_{\mathrm{WIMP}}<10 \mathrm{TeV}$ for the self-annihilation channels WIMP WIMP $\rightarrow \mathrm{b} \overline{\mathrm{b}}$ (green), $\mathrm{W}^{+} \mathrm{W}^{-}$(blue), $\tau^{+} \tau^{-}$(red), $\mu^{+} \mu^{-}$(black), $\nu_{\mu} \bar{\nu}_{\mu}$ (orange). The QFit and $\Lambda$ Fit results are combined.

$$
\rho(\mathrm{r})=\frac{\rho_{\mathrm{s}}}{\left(\mathrm{r} / \mathrm{r}_{\mathrm{s}}\right)\left(1+\mathrm{r} / \mathrm{r}_{\mathrm{s}}\right)^{2}},
$$

with $\mathrm{r}_{\mathrm{s}}=21.7 \mathrm{kpc}$. The normalization of the profile density, $\rho_{\mathrm{s}}$, is computed by fixing the dark matter density at the Sun's position $\rho\left(\mathrm{r}_{\text {Sun }}=8.5 \mathrm{kpc}\right)=0.4 \mathrm{GeV} \cdot \mathrm{cm}^{-3}[43,44]$. The systematic uncertainty introduced by the choice of a specific halo profile is discussed at the end of this section.

The J-Factor can be computed using the package CLUMPY [45] with the contribution from clumps turned off. As demonstrated in Equation 5.3, the J-Factor is a function of the solid angle $\Delta \Omega=2 \pi(1-\cos (\Psi))$. Figure 9 shows the result of the computation of the integrated J-Factor for the NFW profile as a function of $\Psi$. Upper limits for the WIMP velocity-averaged self-annihilation cross-section, $\left\langle\sigma_{\mathrm{A}} \mathrm{v}\right\rangle$, can be set following Equation 5.1 for each benchmark self-annihilation channel, with known J-Factor for a given optimum angular separation $\Psi$ for each $\mathrm{M}_{\mathrm{WIMP}}$, and upper limits $\Phi_{\nu_{\mu}+\bar{\nu}_{\mu}}$ as given in Figure 8. Figure 10 shows the $90 \%$ C.L. upper limits on $\left\langle\sigma_{\mathrm{A}} \mathrm{v}\right\rangle$ as a function of the WIMP mass in the range $25 \mathrm{GeV}<\mathrm{M}_{\text {WIMP }}<10 \mathrm{TeV}$ for the whole set of self-annihilation channels from Expression 3.1.

The $\left\langle\sigma_{\mathrm{A}} \mathrm{v}>\right.$ upper limits obtained from the 2007-2012 ANTARES data are comparable with those obtained by other experiments. Figure 11 shows the resulting $90 \%$ C.L. upper limit on $\left\langle\sigma_{\mathrm{Av}}>\right.$ as a function of the WIMP mass in the range $25 \mathrm{GeV}<\mathrm{M}_{\mathrm{WIMP}}<10 \mathrm{TeV}$ for the self-annihilation channel WIMP WIMP $\rightarrow \tau^{+} \tau^{-}$from this analysis compared to the results from IceCube-DeepCore 79 [46], IceCube 59 [47], and to the most stringent gamma- 


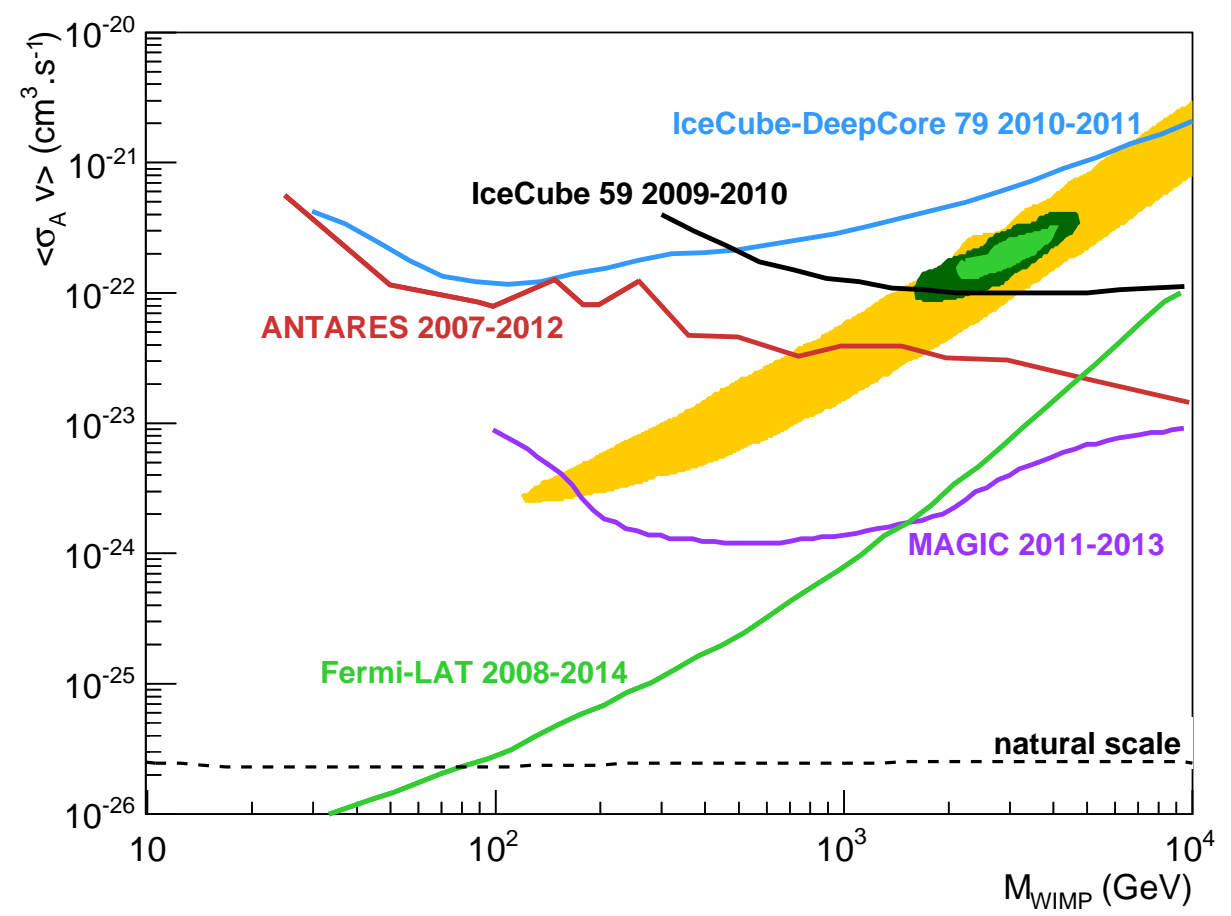

Figure 11. The 90\% C.L. upper limit on the WIMP velocity averaged self-annihilation cross-section, $<\sigma_{\mathrm{AV}}>$, as a function of the WIMP mass in the range $25 \mathrm{GeV}<\mathrm{M}_{\mathrm{WIMP}}<10 \mathrm{TeV}$ for the selfannihilation channel WIMP WIMP $\rightarrow \tau^{+} \tau^{-}$for ANTARES 2007-2012 (red) with QFit and $\Lambda$ Fit results combined. This is compared to the limits from IceCube 59 2009-2010 [47] for the Virgo cluster (black), Fermi-LAT 2008-2014 [48] for the combined analysis of 15 satellite galaxies (green) and MAGIC 2011-2013 [49] for Segue 1 (purple), and the IceCube-DeepCore 79 2010-2011 sensitivity [46] for the GC (blue) is also shown. Interpreting observed electron/positron excesses as dark matter selfannihilations, the orange (PAMELA) and green (PAMELA, Fermi-LAT and H.E.S.S.) ellipses have been obtained [50]. The dashed line indicates the natural scale for which a WIMP is a thermal relic of the early Universe [51].

ray limits from Fermi-LAT [48], and MAGIC [49]. ANTARES 2007-2012 data provides the best upper limit at $90 \%$ C.L. on $\left\langle\sigma_{\mathrm{A}} \mathrm{v}\right\rangle$ for the channel $\tau^{+} \tau^{-}$from a neutrino telescope. Furthermore, the interpretation [50] of the PAMELA excess as a dark matter self-annihilation signature, after being constrained by Fermi-LAT and H.E.S.S., is rejected at 90\% C.L..

All the results are summarised in Tables $1+2$ and $3+4$, where for each WIMP mass and channel the values of the optimised angular separation, $\Psi$, the $90 \%$ C.L. sensitivity, $\bar{\Phi}_{\nu_{\mu}+\bar{\nu}_{\mu}}$, computed from the background without signal expectation, the $90 \%$ C.L. upper limits, $\Phi_{\nu_{\mu}+\bar{\nu}_{\mu}}$, the acceptance, $\overline{\mathrm{A}}_{\mathrm{eff}}\left(\mathrm{M}_{\mathrm{WIMP}}\right) \times \mathrm{T}_{\text {eff }}$, and the $90 \%$ C.L. upper limits on $<\sigma_{\mathrm{A}} \mathrm{v}>$ are presented. In these tables, the results shown in Figures 8 and 10 are highlighted in bold. To evaluate the influence of the dark matter halo profile used in the computation of the $\left\langle\sigma_{\mathrm{A}} \mathrm{v}\right\rangle$ upper limits, different profiles have been tried. The Einasto profile [53], favoured by recent dark matter-only simulations, is given by:

$$
\rho(\mathrm{r})=\rho_{\mathrm{s}} \exp \left\{-(2 / \alpha)\left[\left(\mathrm{r} / \mathrm{r}_{\mathrm{s}}\right)^{\alpha}-1\right]\right\}
$$

where $\mathrm{r}_{\mathrm{s}}=21.7 \mathrm{kpc}$ and $\alpha=0.17$. The isothermal profile [54] is given by: 


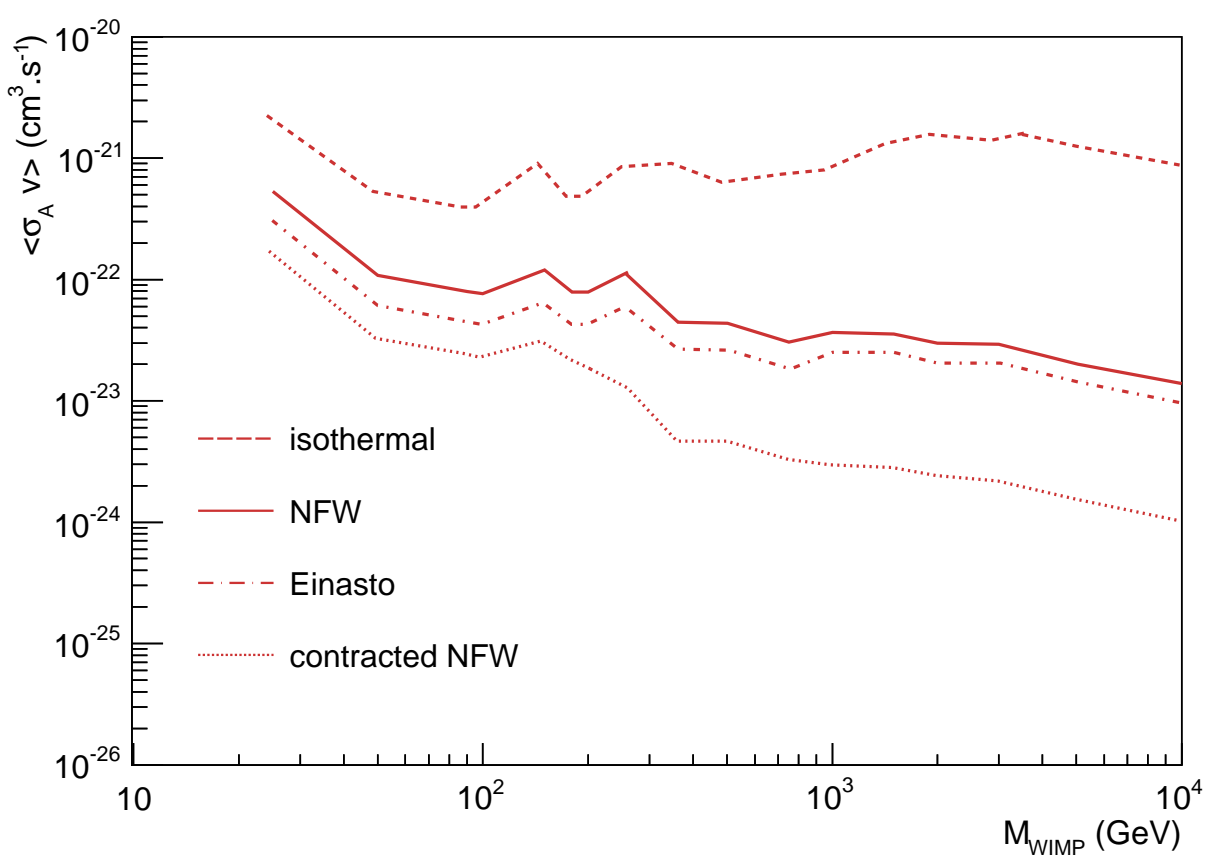

Figure 12. The $90 \%$ C.L. upper limits on the WIMP velocity averaged self-annihilation cross-section, $<\sigma_{\mathrm{AV}}>$, as a function of the WIMP mass in the range $25 \mathrm{GeV}<\mathrm{M}_{\mathrm{WIMP}}<10 \mathrm{TeV}$ for the selfannihilation channel WIMP WIMP $\rightarrow \tau^{+} \tau^{-}$. The dark matter density profiles used in the $\left\langle\sigma_{\mathrm{A}} \mathrm{V}\right\rangle$ computation are NFW [42] (solid line), Einasto [53] (dot-dashed line), isothermal [54] (dashed line) and a contracted NFW [55] (dotted line).

$$
\rho(\mathrm{r})=\frac{\rho_{\mathrm{s}}}{1+\left(\mathrm{r} / \mathrm{r}_{\mathrm{s}}\right)^{2}}
$$

where $\mathrm{r}_{\mathrm{s}}=4 \mathrm{kpc}$. Finally, an adiabatic contraction of the NFW profile due to infall of baryonic matter in the $\mathrm{GC}$ region [55] is given by:

$$
\rho(\mathrm{r})=\frac{\rho_{\mathrm{s}}}{\left(\mathrm{r} / \mathrm{r}_{\mathrm{s}}\right)^{\gamma}\left(1+\mathrm{r} / \mathrm{r}_{\mathrm{s}}\right)^{3-\gamma}}
$$

where $\gamma=1.3$. For all of these profiles, $\rho_{\mathrm{S}}$ is computed as for the NFW profile. Figure 9 summarises the different J-Factors for these profiles. Figure 12 shows the $90 \%$ C.L. upper limit on $\left\langle\sigma_{\mathrm{AV}}\right\rangle$ for the self-annihilation channel WIMP WIMP $\rightarrow \tau^{+} \tau^{-}$for the different dark matter halo profiles. The limits on $\left\langle\sigma_{\mathrm{A}} \mathrm{v}\right\rangle$ for different profiles vary by one to three orders of magnitude depending on the WIMP mass. The optimum angular separation $\Psi$ (Tables 2 and 4) is usually smaller in the high WIMP mass regime, making the computation of the $\left\langle\sigma_{\mathrm{A}} \mathrm{v}\right\rangle$ more sensitive to the cuspiness of a profile for high WIMP masses (Equation 5.3 and Figure 9).

\section{Summary and conclusion}

Using the 2007-2012 data set recorded by the ANTARES neutrino telescope, an indirect search for dark matter towards the Galactic Centre has been performed. The observed number of neutrino events in the Galactic Centre's direction is compatible with the expectation 
from atmospheric backgrounds (Figure 7), for both reconstruction algorithms, optimised for low and high energy events. The $90 \%$ C.L. upper limits have been derived for the neutrino flux, $\Phi_{\nu_{\mu}+\bar{\nu}_{\mu}}$ (Figure 8), and the velocity averaged self-annihilation cross-section, $\left\langle\sigma_{\mathrm{A}} \mathrm{v}\right\rangle$ (Figure 10), for all the self-annihilation channels WIMP WIMP $\rightarrow \mathrm{b} \overline{\mathrm{b}}, \mathrm{W}^{+} \mathrm{W}^{-}, \tau^{+} \tau^{-}, \mu^{+} \mu^{-}$, $\nu_{\mu} \bar{\nu}_{\mu}$, in the range of WIMP masses $25 \mathrm{GeV}<\mathrm{M}_{\text {WIMP }}<10 \mathrm{TeV}$. The $90 \%$ C.L. upper limit on $\left\langle\sigma_{\mathrm{AV}}\right\rangle$ for the channel WIMP WIMP $\rightarrow \tau^{+} \tau^{-}$is the most stringent coming from a neutrino telescope, and is complementary to the most constraining upper limits obtained by the Fermi-LAT and MAGIC observatories (Figure 12). Furthermore, the performance of the ANTARES neutrino telescope through this study allows the rejection at $90 \%$ C.L. of the interpretation [50] of the PAMELA electron/positron excess (constrained by Fermi-LAT and H.E.S.S.) as a signal from dark matter self-annihilation.

\section{Acknowledgments}

The authors acknowledge the financial support of the funding agencies: Centre National de la Recherche Scientifique (CNRS), Commissariat à l'énegie atomique et aux énergies alternatives (CEA), Agence National de la Recherche (ANR), Commission Européenne (FEDER fund and Marie Curie Program), Région Alsace (contrat CPER), Région Provence-Alpes-Côte d'Azur, Département du Var and Ville de La Seyne-sur-Mer, France; Bundesministerium für Bildung und Forschung (BMBF), Germany; Istituto Nazionale di Fisica Nucleare (INFN), Italy; Stichting voor Fundamenteel Onderzoek der Materie (FOM), Nederlandse organisatie voor Wetenschappelijk Onderzoek (NWO), the Netherlands; Council of the President of the Russian Federation for young scientists and leading scientific schools supporting grants, Russia; National Authority for Scientific Research (ANCS), Romania; Ministerio de Ciencia e Innovación (MICINN), Prometeo of Generalitat Valenciana and MultiDark, Spain; Agence de l'Oriental and CNRST, Morocco. We also acknowledge the technical support of Ifremer, AIM and Foselev Marine for the sea operation and the CC-IN2P3 for the computing facilities.

\section{References}

[1] G. Bertone, D. Hooper, J. Silk, Particle Dark Matter: Evidence, Candidates and Constraints, Phys. Rep. 405, 279 (2005).

[2] M. Schumann, Dark Matter 2013, 33rd International Cosmic Ray Conference, Rio de Janeiro 2013, The Astroparticle Physics Conference, [astro-ph/1310.5217].

[3] P. Salati, Dark Matter Annihilation in the Universe, Proceedings of the 2nd International Workshop on Antimatter and Gravity WAG 2013, Bern, Nov. 13-15 2013, [astro-ph/1403.4495].

[4] J. Beringer et al., Particle Data Group, Phys. Rev. D 86, 010001 (2012).

[5] F. Zwicky, Die Rotverschiebung von extragalaktischen Nebeln, Helv. Phys. Acta 6, 110 (1933).

[6] W. J. de Blok, S. S. McGaugh, A. Bosma and V. C. Rubin., Mass density profiles of LSB galaxies, Astrophys. J. 552, L23 (2001).

[7] D. Clowe et al., A direct empirical proof of the existence of dark matter, Astrophys. J. 648, L109 (2006).

[8] P.A.R. Ade et al., Planck Collaboration, Planck 2015 results. XIII. Cosmological parameters, arXiv:1502.01589 [astro-ph.CO] (2015).

[9] E. Komatsu et al., WMAP Collaboration, Seven-Year Wilkinson Microwave Anisotropy Probe (WMAP) Observations: Cosmological Interpretation, Astrophys. J. Suppl. 192, 18 (2011). 
[10] J.R. Ellis et al., Supersymmetric relics from the big bang, Nucl. Phys. B 238, 453 (1984).

[11] D. Hooper, S. Profumo, Dark Matter and Collider Phenomenology of Universal Extra Dimensions, Phys. Rep. 453, 29 (2007).

[12] A. Gould, Direct And Indirect Capture Of Wimps By The Earth, Astrophys. J. 328, 919 (1988); T. K. Gaisser, G. Steigman, and S. Tilav, Limits on Cold Dark Matter Candidates from Deep Underground Detectors, Phys. Rev. D 34, 2206 (1986); J. Silk et al., The Photino, the Sun and High-Energy Neutrinos, Phys. Rev. Lett. 55, 257 (1985); W. H. Press and D. N. Spergel, Capture by the sun of a galactic population of weakly interacting, massive particles, Astrophys. J. 296, 679 (1985).

[13] M. Ageron et al., ANTARES Collaboration, ANTARES: the first undersea neutrino telescope, Nucl. Inst. and Meth. in Phys. Res. A 656, 11 (2011).

[14] P. Amram, ANTARES Collaboration, The ANTARES optical module, Nucl. Inst. and Meth. in Phys. Res. A 484, 369 (2002).

[15] J.A. Aguilar et al., ANTARES Collaboration, Study of large hemispherical photomultiplier tubes for the ANTARES neutrino telescope, Nucl. Inst. and Meth. in Phys. Res. A 555, 132 (2005).

[16] J.A. Aguilar et al., ANTARES Collaboration, Performance of the front-end electronics of the ANTARES Neutrino Telescope, Nucl. Inst. and Meth. in Phys. Res. A 622, 59 (2010).

[17] J.A. Aguilar et al., ANTARES Collaboration, The data acquisition system for the ANTARES neutrino telescope, Nucl. Inst. and Meth. in Phys. Res. A 570, 107 (2007).

[18] P. Amram, ANTARES Collaboration, Sedimentation and Fouling of Optical Surfaces at the ANTARES Site, Astropart. Phys. 19, 253 (2003).

[19] J.A. Aguilar et al., ANTARES Collaboration, AMADEUS - The Acoustic Neutrino Detection Test System of the ANTARES Deep-Sea Neutrino Telescope, Nucl. Inst. and Meth. in Phys. Res. A 626, 128 (2011).

[20] J.A. Aguilar et al., First results of the Instrumentation Line for the deep-sea ANTARES neutrino telescope, ANTARES Collaboration, Astropart. Phys. 26, 314 (2006).

[21] S. Adrián-Martínez et al., The positioning system of the ANTARES Neutrino Telescope, ANTARES Collaboration, JINST 7, T08002 (2012).

[22] M. Ageron et al., ANTARES Collaboration, The ANTARES optical beacon system, Nucl. Inst. and Meth. in Phys. Res. A 578, 498 (2007).

[23] J.A. Aguilar et al., ANTARES Collaboration, Time Calibration of the ANTARES neutrino Telescope, Astropart. Phys. 34, 539 (2011).

[24] J.A. Aguilar et al., ANTARES Collaboration, Search for a diffuse flux of high energy $\nu_{\mu}$ with the ANTARES neutrino telescope, Phys. Lett. B 696, 16 (2011).

[25] S. Adrián-Martínez et al., ANTARES Collaboration, Search for relativistic magnetic monopoles with the ANTARES neutrino telescope, Astropart. Phys. 35, 634 (2012).

[26] S. Adrián-Martínez et al., ANTARES Collaboration, Measurement of atmospheric neutrino oscillations with the ANTARES neutrino telescope, Phys. Lett. B 714, 224 (2012).

[27] S. Adrián-Martínez et al., ANTARES Collaboration, Search for cosmic neutrino point sources with four year data of the ANTARES telescope, Ap. J. Letter 760, 53 (2012).

[28] S. Adrián-Martínez et al., ANTARES Collaboration, Search for muon neutrinos from gamma-ray bursts with the ANTARES neutrino telescope using 2008 to 2011 data, A\&A 559, A9 (2013).

[29] S. Adrián-Martínez et al., ANTARES Collaboration, Measurement of the atmospheric $\nu_{\mu}$ energy spectrum from $100 \mathrm{GeV}$ to $200 \mathrm{TeV}$ with the ANTARES telescope, Eur. Phys. J. C 73, 2606 (2013). 
[30] S. Adrián-Martínez et al., ANTARES Collaboration, First results on dark matter annihilation in the Sun using the ANTARES neutrino telescope, JCAP11, 032 (2013).

[31] S. Adrián-Martínez et al., ANTARES Collaboration, A search for neutrino emission from the Fermi bubbles with the ANTARES telescope, Eur. Phys. J. C 74, 2701 (2014).

[32] S. Adrián-Martínez et al., ANTARES Collaboration, Searches for Point-like and Extended Neutrino Sources Close to the Galactic Centre Using the ANTARES Neutrino Telescope, Ap. J. Letter 786, L5 (2014).

[33] J.A. Aguilar et al., ANTARES Collaboration, A fast algorithm for muon track reconstruction and its application to the ANTARES neutrino telescope, Astropart. Phys. 34, 652 (2011).

[34] M.Cirelli et al., PPPC \& DM ID: A Poor Particle Physicist Cookbook for Dark Matter Indirect Detection, JCAP1103, 051 (2011).

[35] G. Bellini et al., Neutrino oscillations, Advances in High Energy Physics, vol. 2014, Article ID 191960 [hep-ph/1310.7858].

[36] G. Carminati et al., Atmospheric MUons from PArametric formulas: a fast GEnerator for neutrino telescopes (MUPAGE), Comput. Phys. Commun. 179, 915 (2008); M. Bazzotti et al., An update of the generator of atmospheric muons from parametric formulas (MUPAGE), Comput. Phys. Commun. 181, 835 (2010).

[37] G. Barr et al., Flux of atmospheric neutrinos, Phys. Rev. D 39, 3532 (1989); V. Agrawal et al., Atmospheric neutrino flux above 1 GeV, Phys. Rev. D 53, 1314 (1996).

[38] A. Margiotta, Common simulation tools for large volume detectors, Nucl. Instrum. Methods A 725, 53 (2012).

[39] J.A. Aguilar et al., ANTARES Collaboration, Transmission of light in deep sea water at the site of the ANTARES neutrino telescope, Astropart. Phys. 23, 131 (2005).

[40] G.C. Hill, K. Rawlins, Unbiased cut selection for optimal upper limits in neutrino detectors: the model rejection potential technique, Astropart. Phys. 19, 393 (2003).

[41] G.J. Feldman, R.D. Cousins, A Unified Approach to the Classical Statistical Analysis of Small Signals, Phys. Rev. D 57, 3873 (1998).

[42] J.F. Navarro, C.S. Frenk, and S. D. M. White, The Structure of Cold Dark Matter Halos, Ap. J. 462, 563 (1996).

[43] R. Catena and P. Ullio, A novel determination of the local dark matter density, JCAP08, 004 (2010).

[44] P. Salucci, F. Nesti, G. Gentile, and C. Martins, The dark matter density at the Sun's location, A\&A A 83, 523 (2010).

[45] A. Charbonnier, C. Combet, D. Maurin, CLUMPY: A code for $\gamma$-ray signals from dark matter structures, Comp. Phys. Comm. 183, 656 (2012).

[46] M.G. Aartsen et al., IceCube Collaboration, The IceCube Neutrino Observatory Part IV: Searches for Dark Matter and Exotic Particles, 33nd International Cosmic Ray Conference, Rio de Janeiro, 2013 [astro-ph/1309.7007].

[47] M.G. Aartsen et al., IceCube Collaboration, IceCube Search for Dark Matter Annihilation in nearby Galaxies and Galaxy Clusters, Phys. Rev. D88, 122001 (2013).

[48] M. Ackermann et al., Fermi-LAT Collaboration, Searching for Dark Matter Annihilation from Milky Way Dwarf Spheroidal Galaxies with Six Years of Fermi-LAT Data, arXiv:1503.02641 [astro-ph.HE] (2015).

[49] J. Aleksić et al., MAGIC Collaboration, Optimized dark matter searches in deep observations of Segue 1 with MAGIC, JCAP02, 008 (2014). 
[50] P. Meade, M. Papucci, A. Strumia, T. Volansky, Dark Matter Interpretations of the Electron/Positron Excesses after FERMI, Nucl. Phys. B 831, 178 (2010).

[51] G. Steigman, B. Dasgupta, and J.F. Beacom, Precise Relic WIMP Abundance and its Impact on Searches for Dark Matter Annihilation, Phys. Rev. D 86, 023506 (2012).

[52] F. Tegenfeldt, J. Conrad, On Bayesian Treatment of Systematic Uncertainties In Confidence Interval Calculation, Nucl. Inst. and Meth. in Phys. Res. A 539, 407 (2005); J. Conrad et al., Including Systematic Uncertainties in Confidence Interval Construction for Poisson Statistics, Phys. Rev. D 67, 012002 (2003); J. Conrad, Discovery and Upper Limits in Search for Exotic Physics with Neutrino Telescopes, [astro-ph/0612082].

[53] J. F. Navarro et al., The Diversity and Similarity of Simulated Cold Dark Matter Halos, MNRAS 402, 21 (2010).

[54] J. N. Bahcall and R. M. Soneira, The universe at faint magnitudes. I - Models for the galaxy and the predicted star counts, ApJS 44, 73 (1980).

[55] M. Gustafsson, M. Fairbairn, and J. Sommer-Larsen, Baryonic Pinching of Galactic Dark Matter Halos, Phys. Rev. D 74, 123522 (2006). 


\begin{tabular}{|c|c|c|c|c|c|c|c|c|c|c|}
\hline $\begin{array}{r}\mathrm{M}_{\text {WIMP }} \\
(\mathrm{GeV})\end{array}$ & Channel & $\Psi$ & $\bar{\mu}^{90 \%}$ & $\mu^{90}$ & $\begin{array}{c}\overline{\mathrm{A}}_{\mathrm{eff}}\left(\mathrm{M}_{\mathrm{WIMP}}\right) \\
\times \mathrm{T}_{\mathrm{eff}} \\
\left(\mathrm{m}^{2} \cdot \mathrm{yr}\right)\end{array}$ & $\begin{array}{c}\bar{\Phi}_{\nu_{\mu}+\bar{\nu}_{\mu}} \\
\left(\mathrm{km}^{-2} \cdot \mathrm{yr}^{-1}\right)\end{array}$ & $\begin{array}{r}\Phi_{\nu_{\mu}+\bar{\nu}_{\mu}} \\
\left(\mathrm{km}^{-2} \cdot \mathrm{yr}^{-}\right.\end{array}$ & $\begin{array}{l}\mathrm{J}-\text { Factor } \\
\left.\mathrm{GeV}^{2} \cdot \mathrm{cm}^{-5}\right)\end{array}$ & $\begin{array}{l}\overline{<\sigma_{\mathrm{AV}}>} \\
\left(\mathrm{cm}^{3} \cdot \mathrm{s}^{-1}\right)\end{array}$ & $\begin{array}{l}<\sigma_{\mathrm{AV}}> \\
\left(\mathrm{cm}^{3} \cdot \mathrm{s}^{-1}\right)\end{array}$ \\
\hline \multirow[t]{4}{*}{25} & $b \bar{b}$ & 14.6 & 34 & 22 & $1.5 \times 10^{-11}$ & $2.3 \times 10^{18}$ & $1.5 \times 10^{18}$ & $3 \times 10^{22}$ & $2 \times 10^{-19}$ & $1.3 \times 1$ \\
\hline & $\tau \bar{\tau}$ & 14.6 & 34 & 22 & $2.1 \times 10^{-8}$ & $1.6 \times 10^{15}$ & $1.1 \times 10^{15}$ & $3 \times 10^{22}$ & $8.2 \times 10^{-22}$ & $5.3 \times 10^{-22}$ \\
\hline & $\mu^{+} \mu^{-}$ & 14.6 & 34 & 22 & $5.5 \times 10^{-8}$ & $6.2 \times 10^{14}$ & $4 \times 10^{14}$ & $3 \times 10^{22}$ & $7.9 \times 10^{-22}$ & $5.1 \times 10^{-22}$ \\
\hline & $\nu_{\alpha} \bar{\nu}_{\alpha}$ & 10.3 & 30 & 17 & $1.2 \times 10^{-6}$ & $2.5 \times 10^{13}$ & $1.4 \times 10^{13}$ & $2.2 \times 10^{22}$ & $5.8 \times 10^{-23}$ & $3.3 \times 10^{-23}$ \\
\hline \multirow[t]{4}{*}{50} & $b \bar{b}$ & 14.6 & 34 & 22 & $6 \times 10^{-10}$ & $5.7 \times 10^{16}$ & $13.7 \times 10^{16}$ & $3 \times 10^{22}$ & $.6 \times 10^{-20}$ & $1 \times 10^{-20}$ \\
\hline & $\tau \bar{\tau}$ & 12.5 & 32 & 18 & $3.8 \times 10^{-7}$ & $8.5 \times 10^{13}$ & $4.8 \times 10^{13}$ & $2.6 \times 10^{22}$ & $1.9 \times 10^{-22}$ & $1.1 \times 10^{-22}$ \\
\hline & $\mu^{+} \mu^{-}$ & 12.1 & 32 & 21 & $1 \times 10^{-6}$ & $3.1 \times 10^{13}$ & $2.1 \times 10^{13}$ & $2.6 \times 10^{22}$ & $1.9 \times 10^{-22}$ & $1.2 \times 10^{-22}$ \\
\hline & $\nu_{\alpha} \bar{\nu}_{\alpha}$ & 8.95 & 28 & 17 & $1.4 \times 10^{-5}$ & $2 \times 10^{12}$ & $1.2 \times 10^{12}$ & $2 \times 10^{22}$ & $2.1 \times 10^{-23}$ & $10^{-23}$ \\
\hline \multirow[t]{5}{*}{90} & $b \bar{b}$ & 12.5 & 32 & 18 & $5 \times 10^{-9}$ & $6.4 \times 10^{15}$ & $3.6 \times 10^{15}$ & $2.6 \times 10^{22}$ & $5.4 \times 10^{-21}$ & $3 \times 10^{-21}$ \\
\hline & $W^{+} W^{-}$ & 12 & 32 & 20 & $8.6 \times 10^{-8}$ & $3.7 \times 10^{14}$ & $2.3 \times 10^{14}$ & $2.5 \times 10^{22}$ & $3.7 \times 10^{-22}$ & $2.4 \times 10^{-22}$ \\
\hline & $\tau \bar{\tau}$ & 12 & 32 & 20 & $1.9 \times 10^{-6}$ & $1.7 \times 10^{13}$ & $1 \times 10^{13}$ & $2.5 \times 10^{22}$ & $1.3 \times 10^{-22}$ & $8 \times 10^{-23}$ \\
\hline & $\mu^{+} \mu^{-}$ & 12 & 32 & 20 & $5.3 \times 10^{-6}$ & $6 \times 10^{12}$ & $3.8 \times 10^{12}$ & $2.5 \times 10^{22}$ & $1.2 \times 10^{-22}$ & $7.3 \times 10^{-23}$ \\
\hline & $\nu_{\alpha} \bar{\nu}_{\alpha}$ & 9.95 & 29 & 18 & $4.3 \times 10^{-5}$ & $6.8 \times 10^{11}$ & $4.2 \times 10^{11}$ & $2.2 \times 10^{22}$ & $2.1 \times 10^{-23}$ & $1.3 \times 10^{-23}$ \\
\hline \multirow[t]{5}{*}{100} & $b \bar{b}$ & 12.5 & 32 & 18 & & & 2.6 & & & \\
\hline & $W^{+} W^{-}$ & 12 & 32 & 20 & $1.2 \times 10^{-7}$ & $2.7 \times 10^{14}$ & $1.7 \times 10^{14}$ & $2.5 \times 10^{22}$ & $3.4 \times 10^{-22}$ & $2.1 \times 10^{-22}$ \\
\hline & $\tau \bar{\tau}$ & 12 & 32 & 20 & $2.5 \times 10^{-6}$ & $1.3 \times 10^{13}$ & $8.1 \times 10^{12}$ & $2.5 \times 10^{22}$ & $1.2 \times 10^{-22}$ & $7.7 \times 10^{-23}$ \\
\hline & $\mu^{+} \mu^{-}$ & 12 & 32 & 20 & $6.8 \times 10^{-6}$ & $4.7 \times 10^{12}$ & $2.9 \times 10^{12}$ & $2.5 \times 10^{22}$ & $1.1 \times 10^{-22}$ & $7.1 \times 10^{-23}$ \\
\hline & $\nu_{\alpha} \bar{\nu}_{\alpha}$ & 6.95 & 25 & 29 & $4.7 \times 10^{-5}$ & $5.3 \times 10^{11}$ & $6.1 \times 10^{11}$ & $1.6 \times 10^{22}$ & $2.7 \times 10^{-23}$ & $3.2 \times 10^{-23}$ \\
\hline \multirow[t]{5}{*}{150} & $b \bar{b}$ & 12 & 32 & 20 & $2.1 \times 10^{-8}$ & $1.5 \times 10^{15}$ & $10^{14}$ & $2.5 \times 10^{22}$ & $3.1 \times 10^{-21}$ & $10^{-21}$ \\
\hline & $W^{+} W$ & 8.15 & 27 & 21 & $2.8 \times 10^{-7}$ & $9.5 \times 10^{13}$ & $7.4 \times 10^{13}$ & $1.8 \times 10^{22}$ & $3.7 \times 10^{-22}$ & $2.9 \times 10^{-22}$ \\
\hline & $\tau \bar{\tau}$ & 8.15 & 27 & 21 & $5.1 \times 10^{-6}$ & $5.3 \times 10^{12}$ & $4.1 \times 10^{12}$ & $1.8 \times 10^{22}$ & $1.5 \times 10^{-22}$ & $1.2 \times 10^{-22}$ \\
\hline & $\mu^{+} \mu^{-}$ & 8.15 & 27 & 21 & $1.4 \times 10^{-5}$ & $2 \times 10^{12}$ & $1.5 \times 10^{12}$ & $1.8 \times 10^{22}$ & $1.5 \times 10^{-22}$ & $1.1 \times 10^{-22}$ \\
\hline & $\nu_{\alpha} \bar{\nu}_{\alpha}$ & 9.75 & 29 & 18 & $1.1 \times 10^{-4}$ & $2.7 \times 10^{11}$ & $1.7 \times 10^{11}$ & $2.1 \times 10^{22}$ & $2.2 \times 10^{-23}$ & $1.4 \times 10^{-23}$ \\
\hline \multirow[t]{5}{*}{180} & $\overline{b \bar{b}}$ & 12 & 32 & 2 & $3.2 \times 10$ & $1 \times 10^{15}$ & 6.3 & $10^{22}$ & $0^{-21}$ & -21 \\
\hline & $W^{+} W^{-}$ & 9.75 & 29 & 18 & $4.6 \times 10^{-7}$ & $6.3 \times 10^{13}$ & $3.9 \times 10^{13}$ & $2.1 \times 10^{22}$ & $3 \times 10^{-22}$ & $1.8 \times 10^{-22}$ \\
\hline & $\tau \bar{\tau}$ & 9.75 & 29 & 18 & $8.1 \times 10^{-6}$ & $3.6 \times 10^{12}$ & $2.2 \times 10^{12}$ & $2.1 \times 10^{22}$ & $1.3 \times 10^{-22}$ & $7.9 \times 10^{-23}$ \\
\hline & $\mu^{+} \mu^{-}$ & 9.75 & 29 & 18 & $2.1 \times 10^{-5}$ & $1.4 \times 10^{12}$ & $8.5 \times 10^{11}$ & $2.1 \times 10^{22}$ & $1.2 \times 10^{-22}$ & $7.5 \times 10^{-23}$ \\
\hline & $\nu_{\alpha} \bar{\nu}_{\alpha}$ & 9.15 & 28 & 19 & $1.4 \times 10^{-4}$ & $2.1 \times 10^{11}$ & $1.4 \times 10^{11}$ & $2 \times 10^{22}$ & $2.4 \times 10^{-23}$ & $1.6 \times 10^{-23}$ \\
\hline \multirow[t]{5}{*}{200} & $\overline{b \bar{b}}$ & 12 & 32 & 2 & $9^{-8}$ & $10^{14}$ & $10^{14}$ & $10^{22}$ & $10^{-21}$ & $10^{-21}$ \\
\hline & $W^{+} W$ & 9.75 & 29 & 18 & $5.9 \times 10^{-7}$ & $4.9 \times 10^{13}$ & $3 \times 10^{13}$ & $2.1 \times 10^{22}$ & $2.8 \times 10^{-22}$ & $1.7 \times 10^{-22}$ \\
\hline & $\tau \bar{\tau}$ & 9.75 & 29 & 18 & $1 \times 10^{-5}$ & $2.9 \times 10^{12}$ & $1.8 \times 10^{12}$ & $2.1 \times 10^{22}$ & $1.3 \times 10^{-22}$ & $7.9 \times 10^{-23}$ \\
\hline & $\mu^{+} \mu^{-}$ & 9.75 & 29 & 18 & $2.6 \times 10^{-5}$ & $1.1 \times 10^{12}$ & $7 \times 10^{11}$ & $2.1 \times 10^{22}$ & $1.2 \times 10^{-22}$ & $7.5 \times 10^{-23}$ \\
\hline & $\nu_{\alpha} \bar{\nu}_{\alpha}$ & 9.15 & 28 & 19 & $1.7 \times 10^{-4}$ & $1.6 \times 10^{11}$ & $1.1 \times 10^{11}$ & $2 \times 10^{22}$ & $2.2 \times 10^{-23}$ & $1.5 \times 10^{-23}$ \\
\hline \multirow[t]{5}{*}{260} & $\overline{b \bar{b}}$ & 12 & 32 & 20 & $7.2 \times 10^{-8}$ & $4.4 \times 10^{14}$ & $2.8 \times 10^{14}$ & 22 & $10^{-21}$ & $10^{-21}$ \\
\hline & $W^{+} W^{-}$ & 7.85 & 27 & 21 & $9 \times 10^{-7}$ & $3 \times 10^{13}$ & $2.3 \times 10^{13}$ & $1.8 \times 10^{22}$ & $3.4 \times 10^{-22}$ & $2.7 \times 10^{-22}$ \\
\hline & $\tau \bar{\tau}$ & 8.05 & 27 & 20 & $1.5 \times 10^{-5}$ & $1.8 \times 10^{12}$ & $1.3 \times 10^{12}$ & $1.8 \times 10^{22}$ & $1.5 \times 10^{-22}$ & $1.1 \times 10^{-22}$ \\
\hline & $\mu^{+} \mu^{-}$ & 8.05 & 27 & 20 & $3.7 \times 10^{-5}$ & $7.2 \times 10^{11}$ & $5.4 \times 10^{11}$ & $1.8 \times 10^{22}$ & $1.5 \times 10^{-22}$ & $1.1 \times 10^{-22}$ \\
\hline & $\nu_{\alpha} \bar{\nu}_{\alpha}$ & 7.85 & 27 & 21 & $2 \times 10^{-4}$ & $1.3 \times 10^{11}$ & $1 \times 10^{11}$ & $1.8 \times 10^{22}$ & $3.1 \times 10^{-23}$ & $2.4 \times 10^{-23}$ \\
\hline \multirow[t]{5}{*}{360} & $b \bar{b}$ & 10.5 & 30 & 18 & $1.3 \times 10^{-7}$ & $2.3 \times 10^{14}$ & $1.4 \times 10^{14}$ & $2.3 \times 10^{22}$ & $2.2 \times 10^{-21}$ & $1.3 \times 10^{-21}$ \\
\hline & $W^{+} W^{-}$ & 9.25 & 29 & 17 & $1.6 \times 10^{-6}$ & $1.7 \times 10^{13}$ & $1 \times 10^{13}$ & $2 \times 10^{22}$ & $3.1 \times 10^{-22}$ & $1.9 \times 10^{-22}$ \\
\hline & $\tau \bar{\tau}$ & 8.05 & 27 & 20 & $2.6 \times 10^{-5}$ & $1 \times 10^{12}$ & $7.6 \times 10^{11}$ & $1.8 \times 10^{22}$ & $1.6 \times 10^{-22}$ & $1.2 \times 10^{-22}$ \\
\hline & $\mu^{+} \mu^{-}$ & 8.05 & 27 & 20 & $6.2 \times 10^{-5}$ & $4.4 \times 10^{11}$ & $3.2 \times 10^{11}$ & $1.8 \times 10^{22}$ & $1.6 \times 10^{-22}$ & $1.2 \times 10^{-22}$ \\
\hline & $\nu_{\alpha} \bar{\nu}_{\alpha}$ & 9.35 & 29 & 16 & $3.1 \times 10^{-4}$ & $9.2 \times 10^{10}$ & $5.1 \times 10^{10}$ & $2.1 \times 10^{22}$ & $3.1 \times 10^{-23}$ & $1.7 \times 10^{-23}$ \\
\hline
\end{tabular}

Table 1. Results after optimisation from QFit for the angular separation, $\Psi$, the average 90\% C.L. upper limit on the expected signal, $\bar{\mu}^{90 \%}$; the $90 \%$ C.L. upper limit on the expected signal, $\mu^{90 \%}$; the total acceptance, $\overline{\mathrm{A}}_{\mathrm{eff}}\left(\mathrm{M}_{\mathrm{WIMP}}\right) \times \mathrm{T}_{\text {eff }}$; the $90 \%$ C.L. sensitivity on the neutrino flux at Earth, $\bar{\Phi}_{\nu_{\mu}+\bar{\nu}_{\mu}}$; the $90 \%$ C.L. upper limit on the neutrino flux at Earth, $\Phi_{\nu_{\mu}+\bar{\nu}_{\mu}}$; the J-Factor for the given

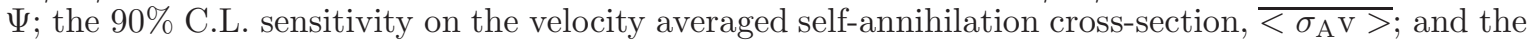
corresponding $90 \%$ C.L. upper limit on $\left\langle\sigma_{\mathrm{AV}}\right\rangle$. Results for $\mathrm{M}_{\mathrm{WIMP}}>360 \mathrm{GeV}$ are available in Table 2. The results shown in Figures 8 and 10 are indicated in bold characters. 


\begin{tabular}{|c|c|c|c|c|c|c|c|c|c|c|}
\hline $\begin{array}{r}\mathrm{M}_{\text {WIMP }} \\
(\mathrm{GeV})\end{array}$ & Channel & $\begin{array}{l}\Psi \\
\left({ }^{\circ}\right)\end{array}$ & $\bar{\mu}^{90 \%}$ & $\mu^{90 \%}$ & $\begin{array}{c}\overline{\mathrm{A}}_{\text {eff }}\left(\mathrm{M}_{\text {WIMP }}\right) \\
\times \mathrm{T}_{\text {eff }} \\
\left(\mathrm{m}^{2} \cdot \mathrm{yr}\right)\end{array}$ & $\begin{array}{c}\bar{\Phi}_{\nu_{\mu}+\bar{\nu}_{\mu}} \\
\left(\mathrm{km}^{-2} \cdot \mathrm{yr}^{-1}\right)\end{array}$ & $\begin{array}{c}\Phi_{\nu_{\mu}+\bar{\nu}_{\mu}} \\
\left(\mathrm{km}^{-2} \cdot \mathrm{yr}^{-1}\right)\end{array}$ & $\begin{array}{c}\mathrm{J}-\text { Factor } \\
\left(\mathrm{GeV}^{2} \cdot \mathrm{cm}^{-5}\right)\end{array}$ & $\begin{array}{l}\overline{<\sigma_{\mathrm{AV}}>} \\
\left(\mathrm{cm}^{3} \cdot \mathrm{s}^{-1}\right)\end{array}$ & $\begin{array}{l}<\sigma_{\mathrm{AV}}> \\
\left(\mathrm{cm}^{3} \cdot \mathrm{s}^{-1}\right)\end{array}$ \\
\hline \multirow[t]{5}{*}{500} & $b \bar{b}$ & 8.15 & 27 & 21 & $2.2 \times 10^{-7}$ & $1.2 \times 10^{14}$ & $9.6 \times 10^{13}$ & $1.8 \times 10^{22}$ & $2.5 \times 10^{-21}$ & $2 \times 10^{-21}$ \\
\hline & $W^{+} W^{-}$ & 9.25 & 29 & 17 & $2.6 \times 10^{-6}$ & $1.1 \times 10^{13}$ & $6.4 \times 10^{12}$ & $2 \times 10^{22}$ & $3.5 \times 10^{-22}$ & $2.1 \times 10^{-22}$ \\
\hline & $\tau \bar{\tau}$ & 9.25 & 29 & 17 & $4.7 \times 10^{-5}$ & $6 \times 10^{11}$ & $3.6 \times 10^{11}$ & $2 \times 10^{22}$ & $1.6 \times 10^{-22}$ & $9.7 \times 10^{-23}$ \\
\hline & $\mu^{+} \mu^{-}$ & 9.25 & 29 & 17 & $1 \times 10^{-4}$ & $2.7 \times 10^{11}$ & $1.6 \times 10^{11}$ & $2 \times 10^{22}$ & $1.6 \times 10^{-22}$ & $9.6 \times 10^{-23}$ \\
\hline & $\nu_{\alpha} \bar{\nu}_{\alpha}$ & 9.95 & 29 & 18 & $4.4 \times 10^{-4}$ & $6.7 \times 10^{10}$ & $4.1 \times 10^{10}$ & $2.2 \times 10^{22}$ & $3.5 \times 10^{-23}$ & $2.1 \times 10^{-23}$ \\
\hline \multirow[t]{5}{*}{750} & $b \bar{b}$ & 8.15 & 27 & 21 & $4.4 \times 10^{-7}$ & $6.1 \times 10^{13}$ & $4.7 \times 10^{13}$ & $1.8 \times 10^{22}$ & $2.5 \times 10^{-21}$ & $1.9 \times 10^{-21}$ \\
\hline & $W^{+} W^{-}$ & 9.05 & 28 & 18 & $5.4 \times 10^{-6}$ & $5.2 \times 10^{12}$ & $3.3 \times 10^{12}$ & $2 \times 10^{22}$ & $3.6 \times 10^{-22}$ & $2.3 \times 10^{-22}$ \\
\hline & $\tau \bar{\tau}$ & 9.05 & 28 & 18 & $9.4 \times 10^{-5}$ & $3 \times 10^{11}$ & $1.9 \times 10^{11}$ & $2 \times 10^{22}$ & $1.8 \times 10^{-22}$ & $1.1 \times 10^{-22}$ \\
\hline & $\mu^{+} \mu^{-}$ & 9.05 & 28 & 18 & $1.9 \times 10^{-4}$ & $1.5 \times 10^{11}$ & $9.3 \times 10^{10}$ & $2 \times 10^{22}$ & $1.8 \times 10^{-22}$ & $1.1 \times 10^{-22}$ \\
\hline & $\nu_{\alpha} \bar{\nu}_{\alpha}$ & 9.05 & 28 & 18 & $8.5 \times 10^{-4}$ & $3.3 \times 10^{10}$ & $2.1 \times 10^{10}$ & $2 \times 10^{22}$ & $3.4 \times 10^{-23}$ & $2.2 \times 10^{-23}$ \\
\hline \multirow[t]{5}{*}{1000} & $b \bar{b}$ & 8.05 & 27 & 20 & $7.1 \times 10^{-7}$ & $3.8 \times 10^{13}$ & $2.8 \times 10^{13}$ & $1.8 \times 10^{22}$ & $2.5 \times 10^{-21}$ & $1.8 \times 10^{-21}$ \\
\hline & $W^{+} W^{-}$ & 8.05 & 27 & 20 & $7.8 \times 10^{-6}$ & $3.4 \times 10^{12}$ & $2.6 \times 10^{12}$ & $1.8 \times 10^{22}$ & $4.4 \times 10^{-22}$ & $3.3 \times 10^{-22}$ \\
\hline & $\tau \bar{\tau}$ & 9.05 & 28 & 18 & $1.5 \times 10^{-4}$ & $1.9 \times 10^{11}$ & $1.2 \times 10^{11}$ & $2 \times 10^{22}$ & $1.9 \times 10^{-22}$ & $1.2 \times 10^{-22}$ \\
\hline & $\mu^{+} \mu^{-}$ & 9.05 & 28 & 18 & $3 \times 10^{-4}$ & $9.3 \times 10^{10}$ & $5.9 \times 10^{10}$ & $2 \times 10^{22}$ & $1.9 \times 10^{-22}$ & $1.2 \times 10^{-22}$ \\
\hline & $\nu_{\alpha} \bar{\nu}_{\alpha}$ & 8.15 & 27 & 21 & $1.1 \times 10^{-3}$ & $2.6 \times 10^{10}$ & $2 \times 10^{10}$ & $1.8 \times 10^{22}$ & $4.5 \times 10^{-23}$ & $3.5 \times 10^{-23}$ \\
\hline \multirow[t]{5}{*}{1500} & $b \bar{b}$ & 8.05 & 27 & 20 & $1.4 \times 10^{-6}$ & $2 \times 10^{13}$ & $1.5 \times 10^{13}$ & $1.8 \times 10^{22}$ & $2.5 \times 10^{-21}$ & $1.9 \times 10^{-21}$ \\
\hline & $W^{+} W$ & 8.15 & 27 & 21 & $1.4 \times 10^{-5}$ & $1.9 \times 10^{12}$ & $1.5 \times 10^{12}$ & $1.8 \times 10^{22}$ & $4.9 \times 10^{-22}$ & $3.8 \times 10^{-22}$ \\
\hline & $\tau \bar{\tau}$ & 8.05 & 27 & 20 & $2.8 \times 10^{-4}$ & $9.8 \times 10^{10}$ & $7.3 \times 10^{10}$ & $1.8 \times 10^{22}$ & $2.4 \times 10^{-22}$ & $1.8 \times 10^{-22}$ \\
\hline & $\mu^{+} \mu^{-}$ & 8.05 & 27 & 20 & $5.2 \times 10^{-4}$ & $5.2 \times 10^{10}$ & $3.9 \times 10^{10}$ & $1.8 \times 10^{22}$ & $2.4 \times 10^{-22}$ & $1.8 \times 10^{-22}$ \\
\hline & $\nu_{\alpha} \bar{\nu}_{\alpha}$ & 10.1 & 30 & 18 & $1.9 \times 10^{-3}$ & $1.5 \times 10^{10}$ & $9.3 \times 10^{09}$ & $2.2 \times 10^{22}$ & $4.1 \times 10^{-23}$ & $2.5 \times 10^{-23}$ \\
\hline \multirow[t]{5}{*}{2000} & $b \bar{b}$ & 8.05 & 27 & 20 & $2.1 \times 10^{-6}$ & $1.3 \times 10^{13}$ & $9.3 \times 10^{12}$ & $1.8 \times 10^{22}$ & $2.6 \times 10^{-21}$ & $2 \times 10^{-21}$ \\
\hline & $W^{+} W^{-}$ & 8.75 & 28 & 18 & $2 \times 10^{-5}$ & $1.4 \times 10^{12}$ & $9.2 \times 10^{11}$ & $1.9 \times 10^{22}$ & $5.6 \times 10^{-22}$ & $3.6 \times 10^{-22}$ \\
\hline & $\tau \bar{\tau}$ & 8.15 & 27 & 21 & $4.1 \times 10^{-4}$ & $6.6 \times 10^{10}$ & $5.1 \times 10^{10}$ & $1.8 \times 10^{22}$ & $2.7 \times 10^{-22}$ & $2.1 \times 10^{-22}$ \\
\hline & $\mu^{+} \mu^{-}$ & 8.15 & 27 & 21 & $7.4 \times 10^{-4}$ & $3.6 \times 10^{10}$ & $2.8 \times 10^{10}$ & $1.8 \times 10^{22}$ & $2.7 \times 10^{-22}$ & $2.1 \times 10^{-22}$ \\
\hline & $\nu_{\alpha} \bar{\nu}_{\alpha}$ & 8.75 & 28 & 18 & $1.9 \times 10^{-3}$ & $1.5 \times 10^{10}$ & $9.5 \times 10^{09}$ & $1.9 \times 10^{22}$ & $7 \times 10^{-23}$ & $4.5 \times 10^{-23}$ \\
\hline
\end{tabular}

Table 2. Extension of Table 1 for $\mathrm{M}_{\text {WIMP }}>360 \mathrm{GeV}$. 


\begin{tabular}{|c|c|c|c|c|c|c|c|c|c|c|}
\hline $\begin{array}{r}\text { MWIMP } \\
(\mathrm{GeV})\end{array}$ & Channel & $\Psi$ & $\bar{\mu}^{90}$ & $\mu^{9}$ & $\begin{array}{c}\overline{\mathrm{A}}_{\mathrm{eff}}\left(\mathrm{M}_{\mathrm{WIMP}}\right) \\
\times \mathrm{T}_{\mathrm{eff}} \\
\left(\mathrm{m}^{2} \cdot \mathrm{yr}\right)\end{array}$ & $\begin{array}{c}\bar{\Phi}_{\nu_{\mu}+\bar{\nu}_{\mu}} \\
\left(\mathrm{km}^{-2} \cdot \mathrm{yr}^{-1}\right)\end{array}$ & $\Phi_{\nu_{\mu}+\bar{\nu}_{\mu}}$ & $\left(\mathrm{GeV}^{2} \cdot \mathrm{cm}^{-5}\right)$ & $\begin{array}{l}\overline{<\sigma_{\mathrm{AV}}>} \\
\left(\mathrm{cm}^{3} \cdot \mathrm{s}^{-1}\right)\end{array}$ & $\begin{array}{l}<\sigma_{\mathrm{AV}}> \\
\left(\mathrm{cm}^{3} \cdot \mathrm{s}^{-1}\right)\end{array}$ \\
\hline \multirow[t]{4}{*}{25} & $b \bar{b}$ & 7.05 & 13 & 14 & $3.1 \times 10^{-13}$ & $4.1 \times 10^{19}$ & $4.4 \times 10^{19}$ & $1.6 \times 10^{22}$ & $6.8 \times 10^{-18}$ & $7.3 \times 10^{-18}$ \\
\hline & $\tau \bar{\tau}$ & 7.05 & 13 & 14 & $5.7 \times 10^{-10}$ & $2.2 \times 10^{16}$ & $2.4 \times 10^{16}$ & $1.6 \times 10^{22}$ & $2.1 \times 10^{-20}$ & $2.2 \times 10^{-20}$ \\
\hline & $\mu^{+} \mu^{-}$ & 7.05 & 13 & 14 & $1.6 \times 10^{-9}$ & $7.9 \times 10^{15}$ & $8.4 \times 10^{15}$ & $1.6 \times 10^{22}$ & $1.9 \times 10^{-20}$ & $2 \times 10^{-20}$ \\
\hline & $\nu_{\mu} \bar{\nu}_{\mu}$ & 7.05 & 13 & 14 & $1.8 \times 10^{-8}$ & $7 \times 10^{14}$ & $7.5 \times 10^{14}$ & $1.6 \times 10^{22}$ & $2.2 \times 10^{-21}$ & $2.4 \times 10^{-21}$ \\
\hline \multirow[t]{4}{*}{50} & $b \bar{b}$ & 14.6 & 28 & 36 & $4 \times 10^{-11}$ & $6.9 \times 10^{17}$ & $9 \times 10^{17}$ & $3 \times 10^{22}$ & $1.9 \times 10^{-19}$ & $2.5 \times 10^{-19}$ \\
\hline & $\tau \bar{\tau}$ & 3.15 & 6 & 7.8 & $8.4 \times 10^{-9}$ & $7.2 \times 10^{14}$ & $9.3 \times 10^{14}$ & $7.5 \times 10^{21}$ & $5.7 \times 10^{-21}$ & $7.5 \times 10^{-21}$ \\
\hline & $\mu^{+} \mu^{-}$ & 3.15 & 6 & 7.8 & $2.2 \times 10^{-8}$ & $2.7 \times 10^{14}$ & $3.5 \times 10^{14}$ & $7.5 \times 10^{21}$ & $5.5 \times 10^{-21}$ & $7.1 \times 10^{-21}$ \\
\hline & $\nu_{\mu} \bar{\nu}_{\mu}$ & 2.45 & 5 & 9.5 & $5.2 \times 10^{-7}$ & $9.7 \times 10^{12}$ & $1.8 \times 10^{13}$ & $5.9 \times 10^{21}$ & $3.3 \times 10^{-22}$ & $6.3 \times 10^{-22}$ \\
\hline \multirow[t]{5}{*}{90} & $b \bar{b}$ & 2.55 & 5.2 & 9 & $2.3 \times 10^{-10}$ & $2.2 \times 10^{16}$ & $3.9 \times 1$ & & $8 \times 10^{-20}$ & $10^{-19}$ \\
\hline & $W^{+} W^{-}$ & 2.55 & 5.2 & 9 & $1.2 \times 10^{-8}$ & $4.5 \times 10^{14}$ & $7.8 \times 10^{14}$ & $6.2 \times 10^{21}$ & $1.9 \times 10^{-21}$ & $3.3 \times 10^{-21}$ \\
\hline & $\tau \bar{\tau}$ & 2.75 & 5.3 & 9.9 & $3 \times 10^{-7}$ & $1.8 \times 10^{13}$ & $3.3 \times 10^{13}$ & $6.7 \times 10^{21}$ & $5.3 \times 10^{-22}$ & $9.8 \times 10^{-22}$ \\
\hline & $\mu^{+} \mu^{-}$ & 2.75 & 5.3 & 9.9 & $8.2 \times 10^{-7}$ & $6.5 \times 10^{12}$ & $1.2 \times 10^{13}$ & $6.7 \times 10^{21}$ & $4.9 \times 10^{-22}$ & $9 \times 10^{-22}$ \\
\hline & $\nu_{\mu} \bar{\nu}_{\mu}$ & 1.85 & 4.2 & 3.5 & $1.2 \times 10^{-5}$ & $3.4 \times 10^{11}$ & $2.9 \times 10^{11}$ & $4.5 \times 10^{21}$ & $5 \times 10^{-23}$ & $4.2 \times 10^{-23}$ \\
\hline \multirow[t]{5}{*}{100} & $\overline{b \bar{b}}$ & 2.55 & 5.2 & 9 & $4 \times 10^{-10}$ & $1.3 \times 10^{16}$ & $2.3 \times 10^{16}$ & $6.2 \times 10^{21}$ & $5.6 \times 10^{-20}$ & $9.7 \times 10^{-20}$ \\
\hline & $W^{+} W^{-}$ & 2.75 & 5.3 & 9.9 & $2.4 \times 10^{-8}$ & $2.2 \times 10^{14}$ & $4.2 \times 10^{14}$ & $6.7 \times 10^{21}$ & $1.1 \times 10^{-21}$ & $2 \times 10^{-21}$ \\
\hline & $\tau \bar{\tau}$ & 2.75 & 5.3 & 9.9 & $5.2 \times 10^{-7}$ & $1 \times 10^{13}$ & $1.9 \times 10^{13}$ & $6.7 \times 10^{21}$ & $3.7 \times 10^{-22}$ & $6.9 \times 10^{-22}$ \\
\hline & $\mu^{+} \mu^{-}$ & 2.75 & 5.3 & 9.9 & $1.4 \times 10^{-6}$ & $3.8 \times 10^{12}$ & $7 \times 10^{12}$ & $6.7 \times 10^{21}$ & $3.5 \times 10^{-22}$ & $6.5 \times 10^{-22}$ \\
\hline & $\nu_{\mu} \bar{\nu}_{\mu}$ & 1.85 & 4.2 & 3.5 & $2.3 \times 10^{-5}$ & $1.8 \times 10^{11}$ & $1.5 \times 10^{11}$ & $4.5 \times 10^{21}$ & $3.3 \times 10^{-23}$ & $2.7 \times 10^{-23}$ \\
\hline \multirow[t]{5}{*}{150} & $b \bar{b}$ & 2.75 & 5.3 & 9.9 & $3.3 \times 10^{-9}$ & $1.6 \times 10^{15}$ & $3 \times 10$ & $.7 \times 10^{21}$ & $1.3 \times 10^{-20}$ & $2.3 \times 10^{-20}$ \\
\hline & $W^{+} W^{-}$ & 3.05 & 5.6 & 9 & $2.3 \times 10^{-7}$ & $2.4 \times 10^{13}$ & $3.9 \times 10^{13}$ & $7.3 \times 10^{21}$ & $2.4 \times 10^{-22}$ & $10^{-22}$ \\
\hline & $\tau \bar{\tau}$ & 2.75 & 5.3 & 9.9 & $3.1 \times 10^{-6}$ & $1.7 \times 10^{12}$ & $3.2 \times 10^{12}$ & $6.7 \times 10^{21}$ & $1.4 \times 10^{-22}$ & $2.6 \times 10^{-22}$ \\
\hline & $\mu^{+} \mu^{-}$ & 2.75 & 5.3 & 9.9 & $8.6 \times 10^{-6}$ & $6.2 \times 10^{11}$ & $1.2 \times 10^{12}$ & $6.7 \times 10^{21}$ & $1.2 \times 10^{-22}$ & $2.3 \times 10^{-22}$ \\
\hline & $\nu_{\mu} \bar{\nu}_{\mu}$ & 1.55 & 3.8 & 2.7 & $7.4 \times 10^{-5}$ & $5.2 \times 10^{10}$ & $3.6 \times 10^{10}$ & $3.8 \times 10^{21}$ & $2.4 \times 10^{-23}$ & $1.7 \times 10^{-23}$ \\
\hline \multirow[t]{5}{*}{180} & $b \bar{b}$ & 2.75 & 5.3 & 9.9 & $6.1 \times 10^{-9}$ & $8.8 \times 10^{14}$ & $1.6 \times 10^{15}$ & $6.7 \times 10^{21}$ & $9.2 \times 10^{-21}$ & $1.7 \times 10^{-20}$ \\
\hline & $W^{+} W$ & 3.05 & 5.6 & 9 & $4.4 \times 10^{-7}$ & $1.3 \times 10^{13}$ & $2 \times 10^{13}$ & $7.3 \times 10^{21}$ & $1.8 \times 10^{-22}$ & $2.8 \times 10^{-22}$ \\
\hline & $\tau \bar{\tau}$ & 3.05 & 5.6 & 9 & $5.7 \times 10^{-6}$ & $1 \times 10^{12}$ & $1.6 \times 10^{12}$ & $7.3 \times 10^{21}$ & $1 \times 10^{-22}$ & $1.7 \times 10^{-22}$ \\
\hline & $\mu^{+} \mu^{-}$ & 3.05 & 5.6 & 9 & $1.5 \times 10^{-5}$ & $3.7 \times 10^{11}$ & $5.9 \times 10^{11}$ & $7.3 \times 10^{21}$ & $9.5 \times 10^{-23}$ & $1.5 \times 10^{-22}$ \\
\hline & $\nu_{\mu} \bar{\nu}_{\mu}$ & 2.85 & 5.4 & 9.7 & $1.8 \times 10^{-4}$ & $3 \times 10^{10}$ & $5.5 \times 10^{10}$ & $6.9 \times 10^{21}$ & $1 \times 10^{-23}$ & $1.9 \times 10^{-23}$ \\
\hline \multirow[t]{5}{*}{200} & $b \bar{b}$ & 2.75 & 5.3 & 9.9 & $9.3 \times 10^{-9}$ & $5.7 \times 10^{14}$ & $1.1 \times$ & $7 \times 10^{21}$ & $10^{-21}$ & $10^{-20}$ \\
\hline & $W^{+} W^{-}$ & 3.05 & 5.6 & 9 & $7.1 \times 10^{-7}$ & $8 \times 10^{12}$ & $1.3 \times 10^{13}$ & $7.3 \times 10^{21}$ & $1.3 \times 10^{-22}$ & $10^{-22}$ \\
\hline & $\tau \bar{\tau}$ & 3.05 & 5.6 & 9 & $8.7 \times 10^{-6}$ & $6.5 \times 10^{11}$ & $1 \times 10^{12}$ & $7.3 \times 10^{21}$ & $8.3 \times 10^{-23}$ & $1.3 \times 10^{-22}$ \\
\hline & $\mu^{+} \mu^{-}$ & 3.05 & 5.6 & 9 & $2.3 \times 10^{-5}$ & $2.4 \times 10^{11}$ & $3.9 \times 10^{11}$ & $7.3 \times 10^{21}$ & $7.6 \times 10^{-23}$ & $1.2 \times 10^{-22}$ \\
\hline & $\nu_{\mu} \bar{\nu}_{\mu}$ & 3.05 & 5.6 & 9 & $2.8 \times 10^{-4}$ & $2 \times 10^{10}$ & $3.3 \times 10^{10}$ & $7.3 \times 10^{21}$ & $7.7 \times 10^{-24}$ & $1.2 \times 10^{-23}$ \\
\hline \multirow[t]{5}{*}{260} & $b \bar{b}$ & 2.75 & 5.3 & 9.9 & $2.9 \times 10^{-8}$ & $1.8 \times 10^{14}$ & $3.4 \times 10^{14}$ & $6.7 \times 10^{21}$ & $3.5 \times 10^{-21}$ & $6.5 \times 10^{-21}$ \\
\hline & $W^{+} W^{-}$ & 2.25 & 4.7 & 9 & $1.6 \times 10^{-6}$ & $3 \times 10^{12}$ & $5.7 \times 10^{12}$ & $5.4 \times 10^{21}$ & $1.1 \times 10^{-22}$ & $2.1 \times 10^{-22}$ \\
\hline & $\tau \bar{\tau}$ & 2.85 & 5.4 & 9.7 & $2.2 \times 10^{-5}$ & $2.4 \times 10^{11}$ & $4.3 \times 10^{11}$ & $6.9 \times 10^{21}$ & $5.5 \times 10^{-23}$ & $9.9 \times 10^{-23}$ \\
\hline & $\mu^{+} \mu^{-}$ & 2.85 & 5.4 & 9.7 & $5.9 \times$ & $9.1 \times 10^{10}$ & $1.6 \times 10^{11}$ & $10^{21}$ & $4.9 \times 10^{-23}$ & $8.9 \times 10^{-23}$ \\
\hline & $\nu_{\mu} \bar{\nu}_{\mu}$ & 1.85 & & 3.5 & $4 \times 10^{-4}$ & $1 \times 10^{10}$ & $8.7 \times 10^{9}$ & $4.5 \times 10^{21}$ & $9.6 \times 10^{-24}$ & $8.1 \times 10^{-24}$ \\
\hline \multirow[t]{5}{*}{360} & $b \bar{b}$ & 2.85 & 5.4 & 9.7 & $1.1 \times 10^{-7}$ & $5.1 \times 10^{13}$ & $9.2 \times 10^{13}$ & $6.9 \times 10^{21}$ & $1.6 \times 10^{-21}$ & $2.9 \times 10^{-21}$ \\
\hline & $W^{+} W^{-}$ & 1.85 & 4.2 & 3.5 & $3.8 \times 10^{-6}$ & $1.1 \times 10^{12}$ & $9.2 \times 10^{11}$ & $4.5 \times 10^{21}$ & $8.9 \times 10^{-23}$ & $7.5 \times 10^{-23}$ \\
\hline & $\tau \bar{\tau}$ & 1.85 & 4.2 & 3.5 & $5 \times 10^{-5}$ & $8.3 \times 10^{10}$ & $7 \times 10^{10}$ & $4.5 \times 10^{21}$ & $5.4 \times 10^{-23}$ & $4.5 \times 10^{-23}$ \\
\hline & $\mu^{+} \mu^{-}$ & 1.85 & 4.2 & 3.5 & $1.3 \times 10^{-4}$ & $3.2 \times 10^{10}$ & $2.7 \times 10^{10}$ & $4.5 \times 10^{21}$ & $4.9 \times 10^{-23}$ & $4.1 \times 10^{-23}$ \\
\hline & $\nu_{\mu} \bar{\nu}_{\mu}$ & 1.85 & 4.2 & 3.5 & $8.8 \times 10^{-4}$ & $4.7 \times 10^{9}$ & $4 \times 10^{9}$ & $4.5 \times 10^{21}$ & $7.2 \times 10^{-24}$ & $6 \times 10^{-24}$ \\
\hline
\end{tabular}

Table 3. Equivalent of Table 1 for $\Lambda$ Fit. 


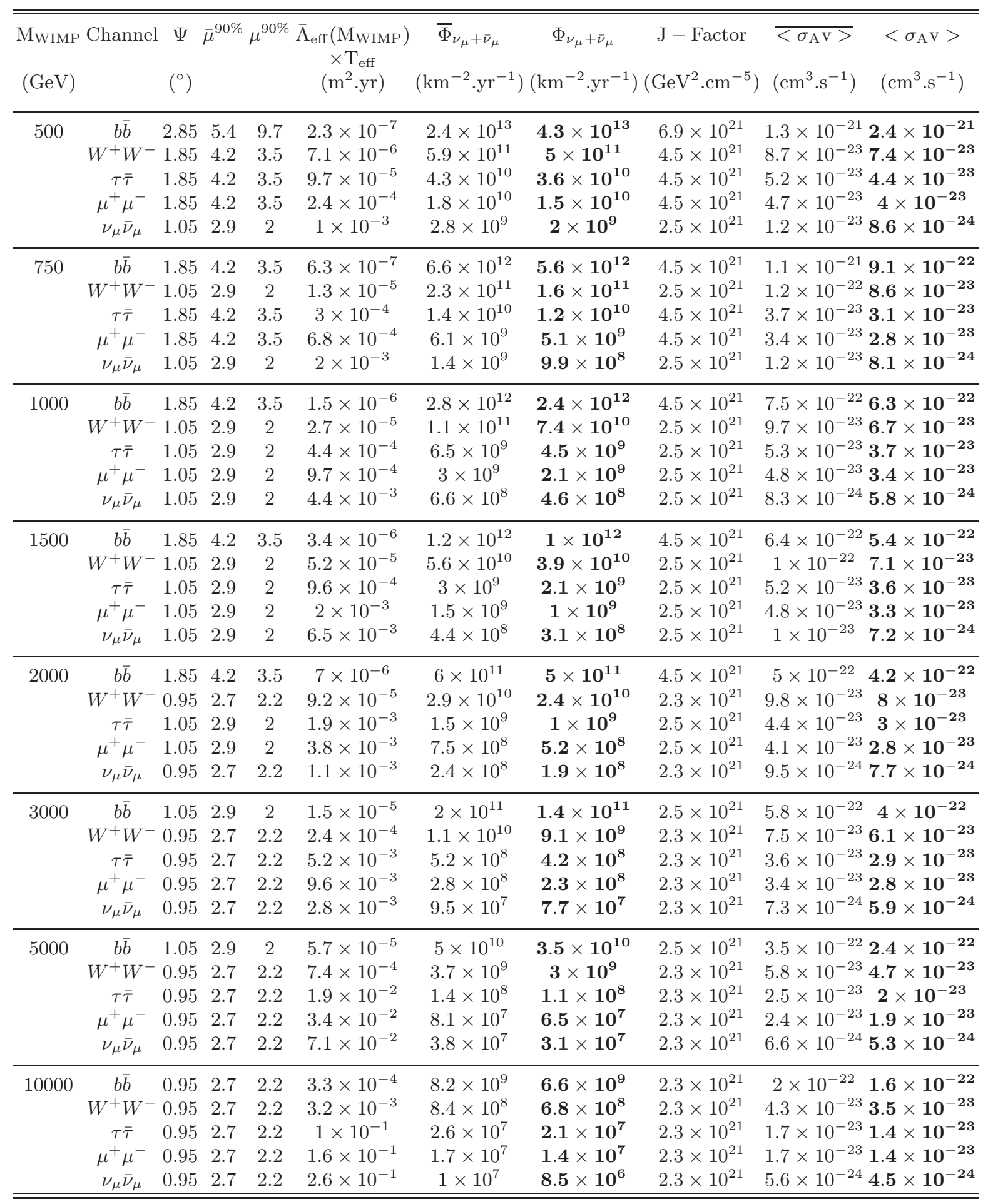

Table 4. Extension of Table 3 for $\mathrm{M}_{\mathrm{WIMP}}>360 \mathrm{GeV}$. 